\title{
ON THE DEFINITION OF THE ESSENTIAL MULTIPLICITY FOR CONTINUOUS TRANS- FORMATIONS IN THE PLANE
}

\author{
BY \\ P. V. REICHELDERFER
}

Chapter I. The question. The result. The method

1.1. Inherent in the definitions of one of the basic concepts in modern theory of the area of a continuous surface (see bibliography) there is a question which this note intends to resolve. The situation will be described presently. Since the question first comes to light in the first two chapters of the paper by Radó and Reichelderfer titled $A$ theory of absolutely continuous transformations in the plane-hereinafter cited as $\mathrm{R}^{2}[1]\left({ }^{1}\right)$ - the reader is requested to consult that paper freely while reading this note. Definitions, theorems, and-as far as practicable-notations in $R^{2}$ [1] will be used in the sequel.

1.2. Let $S$ denote a bounded point set in the $u v$-plane. Given a pair of finite-valued, real-valued functions $x(u, v), y(u, v)$, defined, bounded, and continuous on $S$, these determine a bounded continuous transformation $T: x=x(u, v), y=y(u, v),(u, v) \in S$, from the set $S$ into the $x y$-plane (cf. $\mathrm{R}^{2}$ $[1,1.8])$. For conciseness, complex notation $z=x+i y, w=u+i v$ is introduced, and $T$ is written in the form $T: z=t(w), w \in S$, where $t(w)=x(u, v)+i y(u, v)$, $u+i v=w \in S$, is finite-valued, complex-valued, bounded, continuous on $S$. If $E$ be any set in the $w$-plane, the transformation $T$ associates with each point $z$ in the z-plane a crude multiplicity with respect to $E, N(z, T, E)$, defined to be the number (possibly $+\infty$ ) of distinct points $\mathfrak{w}$ in the set $E \cdot T^{-1}(z)$, where $T^{-1}(\mathfrak{z})$ denotes the set of all points $\mathfrak{w}$ in $S$ for which $t(\mathfrak{w})=\mathfrak{z}$ (cf. $\mathrm{R}^{2}[1$, $1.10,1.8])$.

1.3. Using the crude multiplicity, Banach [1] and Schauder [1] have developed a beautiful, though restrictive, theory of continuous transformations, which has been completed in many respects by Radó [2]. But the crude multiplicity has been found to be generally too large to play an effective role in modern theory of the area. A study of the works of Geöcze led Rado to the idea of replacing the crude multiplicity by a generally smaller multiplicity (see Radó [1]), which has come to be known as the essential multiplicity.

1.4. The intuitive idea of the essential multiplicity is embodied in the following statement.

Definition. Let $T: z=t(w), w \in S$, be a bounded continuous transforma-

Presented to the Society, November 30, 1946; received by the editors September 20, 1946.

(1) Numbers in brackets refer to the bibliography at the end of the paper. 
tion. Assume $E$ is any set in the $w$-plane, and $z$ is any point in the $z$-plane. The $e$-multiplicity $e N(z, T, E)$ of $z$ under the transformation $T$ with respect to the set $E$ is defined as follows. $e N(z, T, E)$ has the finite, nonnegative, integral value $k$ if these conditions are fulfilled: (i) for every positive number $\epsilon$ there exists a bounded continuous transformation $T_{f}: z=t_{f}(w), w \in S$, whose distance $\rho\left(T_{f}, T, S\right)$ from $T$ on $S$ (see $\mathrm{R}^{2}[1,1.9]$ ) is less than $\epsilon$, and for which the crude multiplicity $N\left(z, T_{f}, E\right)$ is equal to $k$; (ii) there exists a positive number $\zeta$ such that every bounded continuous transformation $T_{*}: z=t_{*}(w)$, $w \in S$, whose distance $\rho\left(T_{*}, T, S\right)$ from $T$ on $S$ is less than $\zeta$, has a crude multiplicity $N\left(z, T_{*}, E\right)$ not less than $k . e N(z, T, E)$ has the value $+\infty$ if to every positive in teger $k$ there corresponds a positive number $\delta=\delta(k)$ such that every bounded continuous transformation $T_{*}: z=t_{*}(w), w \in S$, whose distance $\rho\left(T_{*}, T, S\right)$ from $T$ on $S$ is less than $\delta$, has a crude multiplicity $N\left(z, T_{*}, E\right)$ not less than $k$.

1.5. In the literature on surface area and continuous transformations thus far, one of two types of bounded sets $S$ generally serves as the range of definition for a continuous transformation: (i) a bounded finitely connẹcted Jordan region $\Re$ (see $R^{2}[1,1.11]$ ); (ii) a bounded domain $D$ - that is, a bounded connected open set (see $\mathrm{R}^{2}[1,1.13]$ ). Moreover, the set $E$ of 1.4 is restricted to be one of these two types of sets in defining the essential multiplicity (see $\mathrm{R}^{2}[1,1.12,1.13]$ ). But the definitions of the essential multiplicity in the literature are not the one stated in 1.4; the relation between these definitions is to be examined presently.

1.6. First, let $T: z=t(w), w \in \Re$, be any (bounded) continuous transformation, where $\Re$ is a bounded, finitely-connected Jordan region. The reader will readily verify that, for every point $z$ in the $z$-plane, the essential multiplicity $\kappa(z, T, \Re)$ defined in $\mathrm{R}^{2}[1,1.11,1.12]$ has the same value as the $e$-multiplicity $e N(z, T, \Re)$ defined in 1.4. In other words, the definition in 1.4 is an equivalent definition for the essential multiplicity in this case.

1.7. Next, let $T: z=t(w), w \in \mathcal{D}$, be any bounded continuous transformation, where $\mathscr{D}$ is a bounded domain. For any point $z$ in the $z$-plane, the essential multiplicity $\kappa(z, T, \mathcal{D})$ is defined as follows (see $\left.\mathrm{R}^{2}[1,1.13]\right)$. Choose any sequence of finitely-connected Jordan regions $\Re_{n}$ which fill up $\mathcal{D}$ from the interior. Denote by $T_{n}$ the continuous transformation $T_{n}: z=t_{n}(w) \equiv t(w)$, $w \in \Re_{n}$. Then $\kappa\left(z, T_{n}, \Re_{n}\right)$ converges, the limit being possibly $+\infty$; this limit is independent of the choice of the sequence of Jordan regions $\Re_{n}$ which fill up $\mathscr{D}$ from the interior, and is defined to be $\kappa(z, T, \mathcal{D})$. Now $\kappa\left(z, T_{n}, \Re_{n}\right)$ is equal to $e N\left(z, T_{n}, \Re_{n}\right)$ by 1.6 , and it is clear from 1.4 that $e N\left(z, T_{n}, \Re_{n}\right)$ does not exceed $e N(z, T, \mathcal{D})$. Thus $\kappa(z, T, \mathcal{D})$ is never greater than $e N(z, T, \mathcal{D})$. Excepting the case when $\kappa(z, T, \mathscr{D})$ is infinite, it is by no means obvious that $\kappa(z, T, \mathcal{D})$ has the same value as $e N(z, T, \mathcal{D})$.

1.8. Thus the question with which this note is concerned may be phrased as follows (see 1.6). 
Question. Let $\mathcal{D}$ be a bounded domain in the $w$-plane. Given a bounded continuous transformation $T: z=t(w), w \in \mathcal{D}$, consider any point $z$ in the $z$-plane. Is the essential multiplicity $\kappa(z, T, \mathcal{D})$ equal to the $e$-multiplicity $e N(z, T, D)$ defined in 1.4? In other words, do the definitions for essential multiplicity used in the literature differ merely in form, or are two basically different concepts involved?

1.9. In the literature the following characterization theorems are established. ( $\alpha$ ) Assume $T: z=t(w), w \in \Re$, is a (bounded) continuous transformation, where the range of definition is a bounded finitely connected Jordan region $\Re$; for each point $z$ in the $z$-plane, the essential multiplicity $\kappa(z, T, \Re)$ is equal to the number of distinct essential maximal model continua of $z$ under $T$ in the interior $\Re^{0}$ of $\Re$ (see $\mathrm{R}^{2}[1,2.6]$ ). ( $\beta$ ) Assume $T: z=t(w), w \in \mathcal{D}$, is a bounded continuous transformation, where the range of definition is a bounded domain $\mathcal{D}$; for each point $z$ in the $z$-plane, the essential multiplicity $\kappa(z, T, D)$ is equal to the number of distinct essential maximal model continua of $z$ under $T$ in $\mathscr{D}$ (see $\mathrm{R}^{2}[1,1.17]$ ).

1.10. In view of the definition in 1.4 and the characterization theorem in 1.9 , it is clear that the question in 1.8 may be put in the following equivalent form (see 1.7).

Question. Let $\mathcal{D}$ be a bounded domain in the $w$-plane. Given a bounded continuous transformation $T: z=t(w), w \in \mathcal{D}$, if $z$ is a point in the $z$-plane which has exactly $k$ distinct essential maximal model continua under $T$ in $\mathscr{D}$, does there exist, for every positive number $\epsilon$, a bounded continuous transformation $T_{f}: z=t_{f}(w), w \in \mathcal{D}$, whose distance $\rho\left(T_{f}, T, \mathcal{D}\right)$ from $T$ on $\mathcal{D}$ is less than $\epsilon$, and for which the crude multiplicity $N\left(z, T_{f}, \mathcal{D}\right)$ is exactly $k$ ?

1.11. It is the main purpose of this note to answer the questions raised in 1.8 and 1.10 affirmatively by proving the following result.

Theorem. Let $\mathcal{D}$ be a bounded domain in the w-plane. Given a bounded continuous transformation $T: z=t(w), w \in \mathcal{D}$, let $z$ be any point in the $z$-plane and $\epsilon$ any positive number. There exists a bounded continuous transformation $T_{f}: z=t_{f}(w), w \in \mathcal{D}$, such that the distance $\rho\left(T_{f}, T, \mathcal{D}\right)$ is less than $\epsilon$ and the number of models of $z$ under $T_{f}$ in $D$ is equal to the number of essential maximal model continua for $z$ under $T$ in $\mathcal{D}$-that is, $e N(z, T, \mathcal{D})=\kappa(z, T, \mathcal{D})$.

1.12. Another way of putting the matter will be instructive. Retain the assumptions and notations of 1.7. Suppose $\kappa(z, T, \mathcal{D})$ has the finite value $k$. Given a positive number $\epsilon$, let $K_{n}$ denote the class of all bounded continuous transformations $T_{*}: z=t_{*}(w), w \in S$, where the range of definition $S$ contains $\Re_{n}$, for which the distance $\rho\left(T_{*}, T_{n}, \Re_{n}\right)$ is less than $\epsilon$ and the crude multiplicity $N\left(z, T_{*}, \Re_{n}\right)$ is exactly $k$. No $K_{n}$ is empty (see 1.6). It is to be shown that there is a continuous transformation $T_{\boldsymbol{t}}$ belonging to $K_{n}$ for every integer $n$.

1.13. In chapter II, two important tools are developed for making a proof of the theorem in 1.11. There is a process for welding two continuous trans- 
formations together under suitable conditions; for a precise statement of this process, the reader may consult 2.11. A method for approximating to a continuous transformation by a quasi-polyhedral transformation (see 2.13) is then developed (see $2.14,2.16$ ). Similar approximations have been used in the literature (see $\mathrm{R}^{2}$ [1, footnote 30 ], for example), but not in a form sufficiently refined for the purpose needed here.

1.14. The components $\sigma$ of $T^{-1}(z)$ are of two types (see 3.1): (i) $\sigma$ may be a continuum-a closed connected set in $\mathcal{D}$; (ii) $\sigma$ may not be a continuum, in which case its closure contains a point not in $\mathcal{D}$. Components of the first type are immersed in connected open sets $g$ in $\mathcal{D}$ whose boundaries contain no points of $T^{-1}(z)$ and whose images under $T$ lie arbitrarily close to $z$ (see 3.2, 3.3). Inside each $g$ the transformation $T$ is modified slightly in a continuous way so that $z$ has at most one model in $g$ under the modified transformation, and if it has a model under the modified transformation in $g$, then $z$ has an essential maximal model continuum under $T$ in $g$ (see 3.8). A first modification $T$ of $T$ results, as described in chapter III, in which all the components of $T^{-1}(z)$ of the first type are replaced by a number of isolated points in $T_{\vec{f}}(z)$ not exceeding the number of essential maximal model continua for $z$ under $T$ in $\mathcal{D}$, while the components of $T_{t^{-1}}^{-1}(z)$ of the second type are identical with the components of the second type of $T^{-1}(z)$. The reader may inspect 3.13 for a more detailed statement of this result.

1.15. In chapter IV, attention is restricted to a point $z$ for which all the components $\sigma$ of $T^{-1}(z)$ are of the second type (see 1.14). It is proven that there are continuous transformations $T_{f}$ arbitrarily close to $T$ such that $z$ has no models under $T_{t}$ in $\mathcal{D}$ (see 4.65). The idea behind the proof is simple. Consider, for a moment, a continuous transformation $T_{*}: z=t_{*}(w)$, $w \in \Re$, where the range of definition is a finitely connected Jordan region $\Re$. If $\sigma_{*}$ is any component of $T_{*}^{-1}(z)$ which meets the boundary of $\Re$, then $T_{*}$ may be modified slightly in a neighborhood of $\sigma_{*}$ so that $z$ has no models under the modified transformation in a vicinity of $\sigma_{*}$ (cf. 1.6). If one chooses a sequence of finitely-connected Jordan regions $\Re_{n}$ which fill up $\mathcal{D}$ from the interior, one may hope to apply this technique to each transformation $T_{n}: z=t_{n}(w) \equiv t(w)$, $w \in \Re_{n}$, in turn, to obtain a sequence of modified transformations converging to a transformation $T_{\boldsymbol{f}}$ on $\mathcal{D}$ under which $z$ has no models in $\mathcal{D}$. This is the idea in chapter IV, but care must be exercised, for a component $\sigma$ of $T^{-1}(\mathrm{z})$ of the second type may meander in and out of a Jordan region $\Re$ in $\mathcal{D}$.

1.16. In chapter $V$, the results of chapters II, III, IV are synthesized to make a proof for the theorem in 1.11 .

1.17. In addition to notations used in $R^{2}$ [1], the following are adopted in the sequel. Let $E$ be any set in the $w$-plane. Then $\mathcal{C} E$ will denote the complement of $E$-that is, the set of all points $w$ which are not in $E$. The set of all points which either belong to $E$ or are limit points of $E$ - that is, the closure of $E$-will be indicated by $C E$. The frontier of $E, C E \cdot C C E$, will be repre- 
sented by fr $E$; the interior of $E, \mathcal{C} C \mathcal{C}$, will be denoted by $E^{0}$. If $f(w)$ be any finite-valued, complex-valued function defined on $E$, then $M(f, E)$ will denote the least upper bound of $|f(w)|$ for $w$ in $E$, and $m(f, E)$ will denote the greatest lower bound of $|f(w)|$ for $w$ in $E$. If $\delta$ be any positive number, then $O(\delta, f, E)$ will denote the least upper bound of $\left|f\left(w^{\prime}\right)-f\left(w^{\prime \prime}\right)\right|$ for points $w^{\prime}, w^{\prime \prime}$ in $E$ such that $\left|w^{\prime}-w^{\prime \prime}\right|$ does not exceed $\delta$ (cf. $\left.\mathrm{R}^{2}[1,2.3]\right)$. Finally, $|E|$ will denote the exterior measure of the set $E$.

\section{Chapter II. A welding process. An approximation lemma}

2.1. A directed line segment in the $w$-plane with first end point $a$ and second end point $b$ will be denoted by $a b$. If $a b$ and $A B$ are two directed line segments, then $\Delta$, or $\Delta(a, b, A, B)$, will denote the greater of the distances $|A-a|$ and $|B-b|$. In case the lines to which $a b$ and $A B$ belong are parallel and at a distance $\delta$ from each other, it is clear that $\Delta$ is not less than $\delta$; also $\Delta$ and $\delta$ are equal if and only if $a b$ and $A B$ have the same direction and each is the orthogonal projection of the other.

2.2. Suppose that $a b$ and $A B$ are directed line segments belonging to two distinct parallel lines and having the same direction. Then the two lines determined by the points $a, A$ and $b, B$ respectively are distinct and have exactly one point $O$ in common, which will be the point at infinity if these lines are parallel, and which does not lie in the closed trapezoid $a b B A$. A correspondence $\mathbb{S}$ between points $p$ on $a b$ and points $P$ on $A B$ is defined as follows: $p \subseteq P$ if and only if the line determined by $p$ and $P$ passes through $O$. It is readily seen that this correspondence $\mathbb{E}$ has the following properties: $(\alpha) a \mathbb{S} A$, $b \mathbb{E} B ;(\beta) \mathbb{E}$ is biunique and bicontinuous, hence topological; $(\gamma)$ if $p \mathfrak{S} P$ then $|P-p|$ does not exceed $\Delta ;(\delta)$ if $w$ is any point in the closed trapezoid $a b B A$ there is uniquely determined a point $p$ on $a b$ and a point $P$ on $A B$ such that $p \subseteq P$ and $w$ lies on the line segment $p P$. In particular, if $w$ is on $a b$ then $w$ and $p$ coincide, while if $w$ is on $A B$ then $w$ and $P$ coincide.

2.3. In the sequel $\mathfrak{p}$, or $\mathfrak{P}$, will be the generic symbol for a nondegenerate simple closed polygon in the $w$-plane. A line segment in $\mathfrak{p}$ which is a proper subset of no other line segment in $\mathfrak{p}$ is termed a side of $\mathfrak{p}$. Two sides of $p$ either have no points in common, or an end point in common -in the latter case the sides are said to be adjacent and their common end point is termed a vertex of $\mathfrak{p}$. Every vertex of $\mathfrak{p}$ is the common end point of exactly two sides-these sides are adjacent and not parallel. The number of vertices of $\mathfrak{p}$ is equal to the number of sides of $\mathfrak{p}$. If every side of $\mathfrak{p}$ is parallel either to the $u$-axis or to the $v$-axis, then $p$ will be termed an oriented polygon. Of two adjacent sides of an oriented polygon it is clear that one must be parallel to the $u$-axis and the other must be parallel to the $v$-axis. An oriented polygon has an even number of sides and vertices. To simplify proofs attention is restricted to oriented polygons. However, the results to be established presently for oriented polygons may be verified under much more general hypotheses. 
2.4. Suppose that $\mathfrak{p}$ and $\mathfrak{P}$ are two oriented polygons in the $w$-plane satisfying the following conditions: (i) $\mathfrak{p}$ and $\mathfrak{B}$ bound a closed polygonal region $\Re$ in the $w$-plane; (ii) $\mathfrak{p}$ and $\mathfrak{B}$ each have the same number $n$ of sides and of vertices; (iii) notations $a_{1}, \cdots, a_{n}$ and $A_{1}, \cdots, A_{n}$ for the vertices of $\mathfrak{p}$ and of $\mathfrak{P}$ respectively may be chosen so that the directed segments $a_{j-1} a_{j}$ and $A_{j-1} A_{j}$ are sides of $\mathfrak{p}$ and $\mathfrak{P}$ respectively and have the same direction for $j$ between 1 and $n$-where $a_{0}$ and $A_{0}$ are defined to be $a_{n}$ and $A_{n}$ respectively; (iv) the line segments $a_{j} A_{j}$ for $j$ between 1 and $n$ are contained in $\Re$ and divide $\Re$ in to exactly $n$ nonoverlapping simply connected polygonal regions-each of which is a trapezoid. Let $\Delta(\mathfrak{p}, \mathfrak{B})$ denote the maximum of $\Delta\left(a_{j-1} a_{j}, A_{j-1}, A_{j}\right)$ for $j$ between 1 and $n$ (see 2.1). A correspondence $\mathfrak{E}$ between points $p$ on $\mathfrak{p}$ and points $P$ on $\mathfrak{B}$ is defined as follows: $p \Subset P$ if and only if there exists a $j$ between 1 and $n$ such that $p$ belongs to $a_{j-1} a_{j}$ and $P$ belongs to $A_{j-1} A_{j}$, and $p \mathbb{C} P$ in the sense defined in 2.2. It is readily verified that this correspondence $\mathbb{S}$ has the

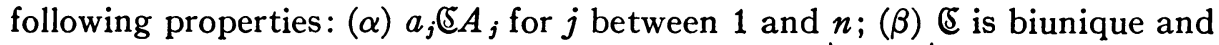
bicontinuous, hence topological; $(\gamma)$ if $p \Subset P$ then $|P-p|$ does not exceed $\Delta(\mathfrak{p}, \mathfrak{P}) ;(\delta)$ if $w$ is any point in the closed polygonal region $\Re$ there is uniquely determined a point $p$ on $p$ and a point $P$ on $\mathfrak{B}$ such that $p \mathfrak{S} P$ and $w$ lies on the line segment $p P$. In particular, if $w$ is on $p$ then $w$ and $p$ coincide, while if $w$ is on $\mathfrak{P}$ then $w$ and $P$ coincide.

2.5. Let $\mathfrak{B}$ be any oriented polygon in the $w$-plane, let 2 be any open set containing $\mathfrak{B}$, and let $\sigma$ be any positive number. Elementary considerations show that there always exists a polygon $\mathfrak{p}$ in $2-$ necessarily orientedsuch that $\mathfrak{p}$ and $\mathfrak{P}$ satisfy the conditions enumerated in 2.4 and $\Delta(\mathfrak{p}, \mathfrak{P})$ is less than $\sigma$. Furthermore, $p$ may be chosen either in the in terior of the bounded region determined by $\mathfrak{B}$ or in the exterior of that region.

2.6. Suppose that $\mathfrak{p}$ and $\mathfrak{B}$ are two oriented polygons satisfying the conditions enumerated in 2.4 ; let $\Re$ and $\Delta(\mathfrak{p}, \mathfrak{B})$ have the meanings assigned there. Assume there is given (i) a single-valued, finite-valued, complex-valued, function $f(w), w \in \Re$, defined and continuous in $\Re$ and (ii) a function $F(w), w \in \mathfrak{B}$, defined and continuous on $\mathfrak{B}$. For every $w$ in $\Re$ there is uniquely determined a point $p$ on $p$ and a point $P$ on $\mathfrak{B}$ such that $p \mathfrak{S} P$ and $w$ lies on the line segment $p P$ (see $2.4(\delta))$. Define

$$
g(w)=f(p) \frac{|P-w|}{|P-p|}+F(P) \frac{|w-p|}{|P-p|}, \quad w \in \Re .
$$

It is easy to verify that the function $g(w), w \in \Re$, has the following properties: $(\alpha) g$ is defined, single-valued, finite-valued, complex-valued, continuous in $\Re$; $(\beta) \mathrm{g}$ reduces to $f$ on $\mathfrak{p} ;(\gamma) g$ reduces to $F$ on $\mathfrak{P} ;(\delta) M(g-f, \Re)$ does not exceed $M(F-f, \mathfrak{B})+O[\Delta(\mathfrak{p}, \mathfrak{B}), f, \Re]$ (see 1.17).

2.7. Now let $\mathscr{D}$ be any bounded domain in the $w$-plane, and suppose $\mathfrak{B}$ is any oriented (simple closed) polygon in $\mathcal{D}$-the interior of $\mathfrak{B}$ need not lie in $\mathscr{D}$. Then $\mathfrak{B}$ divides $\mathcal{D}$ into two domains and constitutes part of the bound- 
ary of each (see Newman $[1, \mathrm{~V}]$ ) - let $G$ denote either one of these. Assume there is given (i) a function $f(w), w \in G+\mathfrak{B}$, dęfined and continuous in $G+\mathfrak{B}$, (ii) a function $F(w), w \in \mathfrak{B}$, defined and continuous on $\mathfrak{B}$, (iii) a complex constant $z$ such that $m(F-z, \mathfrak{B})$ exceeds $M(F-f, \mathfrak{B})$ (see 1.17), and (iv) a positive constant $\epsilon$. Since condition (iii) implies that $f$ does not take on the value $z$ on $\mathfrak{B}$, an open set 2 containing $\mathfrak{B}$ may be chosen in $\mathcal{D}$ so that $f$ is different from $z$ in $G$ Q. Let $\delta$ denote the smaller of the positive constants $\epsilon$ and $m(F-z, \mathfrak{B})$ $-M(F-f, \mathfrak{B})$. In view of the uniform continuity of the continuous function $f$ on any closed set in $G+\mathfrak{B}$, there follows the existence of a positive constant $\sigma$ such that, if $E$ is any set in $G+\Re$ each point of which is within $\sigma$ of some point on $\mathfrak{B}$, then $O[\sigma, f, E]$ is less than $\delta$. From 2.5 it follows that there exists an oriented polygon $\mathfrak{p}$ in $G \mathcal{Q}$ such that $\mathfrak{p}$ and $\mathfrak{B}$ satisfy the conditions enumerated in $2.4, \Re$ is contained in 2 , and $\Delta(\mathfrak{p}, \mathfrak{B})$ is less than $\sigma$. Clearly $O[\Delta(\mathfrak{p}, \mathfrak{B}), f, \Re]$ is less than $\delta$. Consider the function $g(w), w \in \Re$, defined in 2.6 ; in addition to the properties $(\alpha)-(\delta)$ listed there, it is easily seen to satisfy the following conditions: $(\xi) M(g-f, \Re)$ is less than $M(F-f, \mathfrak{B})+\epsilon ;(\eta)$ $m(g-\mathfrak{z}, \Re)$ is not less than the positive constant

$$
m(F-\mathfrak{z}, \mathfrak{B})-M(F-f, \mathfrak{B})-O[\Delta(\mathfrak{p}, \mathfrak{B}), f, \mathfrak{R}] ;
$$

consequently $g$ does not assume the value $z$ on $\Re$.

2.8. Given a bounded finitely-connected Jordan region $\Re$ in the $w$-plane, $\Re$ is termed a polygonal region if each boundary curve of $\Re$ is a nondegenerate simple closed polygon (see 2.3 ). If, moreover, each boundary curve of $\Re$ is an oriented polygon, then $\Re$ is termed an oriented polygonal region.

2.9. Again, let $\mathcal{D}$ be any bounded domain in the w-plane, and suppose that $\Re$ is any finitely-connected oriented polygonal region in $\mathcal{D}$. Assume there is given (i) a bounded continuous transformation $T: z=t(w), w \in \mathcal{D C} \Re^{0}$ (see

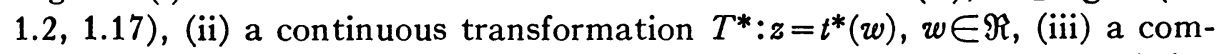
plex constant $z$ such that $m\left(t^{*}-z, \Re C \Re^{0}\right)$ exceeds $M\left(t^{*}-t, \Re C \Re^{0}\right)$, and (iv) a positive constant $\epsilon$. Since condition (iii) implies that $t$ does not take on the value $z$ on $\Re \mathcal{C} \Re^{0}$, an open set 2 containing $\Re$ may be chosen in $\mathcal{D}$ so that $t$ is different from $z$ in $2 \mathcal{C} \Re^{0}$. In view of $2.5-2.7$ it is clear that there exists an oriented polygonal region $\mathfrak{r}$ in $\Phi$ containing $\Re$ in its interior $\mathfrak{r}^{0}$ and such that $\mathfrak{r} C \Re^{0}$ is contained in 2 , and a function $g(w), w \in \mathfrak{r} C \Re^{0}$, which satisfies the following conditions: $(\alpha) g$ is defined, single-valued, finite-valued, complexvalued, continuous in $\mathfrak{r} \mathcal{C} \Re^{0} ;(\beta) g$ reduces to $t$ on $\mathfrak{r} \mathcal{C} \mathfrak{r}^{0} ;(\gamma) g$ reduces to $t^{*}$ on $\Re \mathcal{A} \Re^{0} ;(\delta) M\left(g-t, \mathfrak{r} C \Re^{0}\right)$ is less than $M\left(t^{*}-t, \Re C \Re^{0}\right)+\epsilon ;(\epsilon) g$ does not assume the value $z$ on $\mathfrak{r} C \Re^{\circ}$.

2.10. Retain the assumptions and conclusions of 2.9. Define

$$
\left.T_{*}: \quad z=t_{*} w\right) \equiv\left\{\begin{array}{lr}
t^{*}(w), & w \in \Re, \\
g(w), & w \in \mathfrak{r} \mathcal{C} \Re^{0}, \quad w \in \mathcal{D}, \\
t(w), & w \in \mathcal{D C} \mathfrak{r}^{0} .
\end{array}\right.
$$


Evidently the transformation $T_{*}$ possesses the following properties: $(\alpha) T_{*}$ is a bounded continuous transformation in $\mathcal{D} ;(\beta) T_{*}$ is identical with $T^{*}$ in $\Re$; $(\gamma) T_{*}$ is identical with $T$ in $\mathcal{D C Q} ;(\delta) \rho\left(T_{*}, T, \mathcal{D} \mathcal{C} \Re^{0}\right)$ is less than

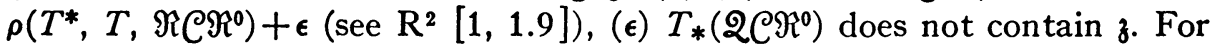
brevity, the transformation $T_{*}$ is termed a weld of the transformation $T^{*}$ in $\Re$ to the transformation $T$ outside the open set 2 containing $\Re$ with a tolerance of $\epsilon$ and the exclusion of the value $z$ in $T_{*}\left(2 \bigodot \Re^{0}\right)$. Of course, $T_{*}$ is not unique.

2.11. The preceding results are summarized in this statement.

Welding LEMma. Let $\mathcal{D}$ be any bounded domain in the w-plane; suppose that $\Re$ is any finitely connected oriented polygonal region in $\mathcal{D}$, and $\epsilon$ is any positive constant. Assume there is given a bounded continuous transformation $T: z=t(w), w \in \mathcal{D} \Re^{0}$, and a continuous transformation $T^{*}: z=t^{*}(w), w \in \Re$. Let $z$ be any point for which $m\left(t^{*}-z, \Re C \Re^{0}\right)$ exceeds $M\left(t^{*}-t, \Re \bigodot \Re^{0}\right)$. Then an open set 2 containing $\Re$ may always be chosen in $\mathcal{D}$ so that $T\left(2 \mathcal{C} \Re^{0}\right)$ does not contain 8 . There exists a weld $T_{*}$ of $T^{*}$ in $\Re$ to $T$ outside of 2 with a tolerance of $\epsilon$ and the exclusion of the value $z$ in $T_{*}\left(2 \bigodot \Re^{0}\right)$.

2.12. Let $\mathcal{D}$ be any bounded domain in the $w$-plane. It can be shown (cf. Newman $[1, V I])$ that there exists a sequence of finitely connected Jordan regions $\Re_{n}$ satisfying the following conditions: (i) $\Re_{n}$ is contained in the interior $\Re_{n+1}^{0}$ of $\Re_{n+1}$ for every $n$; (ii) the point set sum of the $\Re_{n}$ is identical with $\mathscr{D}$; (iii) each $\Re_{n}$ is an oriented polygonal region (see 2.8 ). It is readily seen that conditions (i) and (ii) alone imply that the $\Re_{n}$ fill up $\mathcal{D}$ from the interior (see $\left.\mathrm{R}^{2}[1,1.13]\right)$. Indeed, if $F$ is any closed set in $D$ it follows that for every $w$ in $F$ there exists an $n(w)$ such that $w$ is contained in $\Re_{n(w)}^{0}$. The family of open sets $\Re_{n(w)}^{0}, w \in F$, covers the bounded closed set $F$, and the Borel covering theorem shows that a finite number of these $\Re_{n(w)}^{0}$ covers $F$. If $n_{0}$ denotes the largest integer appearing among the $n(w)$ of this finite subset, then clearly $F$ is contained in $\Re_{n}$ for every $n$ exceeding $n_{0}$. Moreover, it is clear that any infinite subsequence of a sequence of Jordan regions $\Re_{n}$ satisfying conditions (i) and (ii) also satisfies conditions (i) and (ii). Finally, if $G$ is any bounded connected open set - that is, a bounded domain - and $F$ is any closed set in $G$, there exists a finitely connected Jordan region $\Re$ which is an oriented polygonal region in $G$ and which contains $F$ in its interior $\Re^{0}$.

2.13. Let $\mathcal{D}$ be a bounded domain in the w-plane. A continuous transformation $T: z=t(w), w \in \mathcal{D}$, is said to be quasi-polyhedral if there exists a sequence of finitely connected Jordan regions $\Re_{n}$ such that the following conditions are satisfied: (i) $\Re_{n}$ is contained in the interior $\Re_{n+1}^{0}$ of $\Re_{n+1}$ for every $n$; (ii) the point set sum of the $\Re_{n}$ is identical with $\mathscr{D}_{\text {; }}$ (iii) each $\Re_{n}$ is a polygonal region (see 2.8); (iv) the continuous transformation $T_{n}: z=t_{n}(w) \equiv t(w), w \in \Re_{n}$, is polyhedral - that is, there exists a rectilinear triangulation $\mathcal{T}_{n}$ of $\Re_{n}$ (a subdivision of $\Re_{n}$ in to a finite number of nonoverlapping, nondegenerate recti- 
linear triangles $\delta_{n}$, any two of which have either no point in common, a vertex in common, or an entire side in common) such that $T_{n}$ maps each rectilinear triangle $\delta_{n}$ in $\mathcal{G}_{n}$ topologically onto a nondegenerate rectilinear triangle $\Delta_{n}$ in the z-plane, carrying the vertices of $\delta_{n}$ onto the vertices of $\Delta_{n}$. Observe that conditions (i) and (ii) imply that the $\Re_{n}$ fill up $D$ from the interior (see 2.12). Clearly, if $F$ is any closed set in $D$ and $z$ is any point in the $z$-plane, then the number of models of $z$ under $T$ in $F, N(z, T, F)$, is finite; consequently the number of models of $z$ under $T$ in $\mathcal{D}, N(z, T, \mathscr{D})$, is at most enumerable (see $\mathrm{R}^{2}[1,1.10]$ ).

2.14. Approximation lemma. Let $T: z=t(w), w \in \mathcal{D}$, be any bounded continuous transformation (see 1.2), where $D$ is a bounded domain. If $\epsilon$ is any positive number there exists a quasi-polyhedral transformation $T_{c}: z=t_{e}(w), w \in \mathcal{D}$, such that the distance $\rho\left(T_{\epsilon}, T, \mathcal{D}\right)$ is less than $\epsilon$. Indeed, more will be shown. Choose any sequence of finitely connected Jordan regions $\Re_{n}$ satisfying conditions (i), (ii), (iii) in 2.13, and any sequence of positive numbers $\epsilon_{n}$. Then there exists a quasi-polyhedral transformation $T_{*}: z=t_{*}(w), w \in \mathcal{D}$, such that the distance $\rho\left(T_{*}, T, \Re_{n} \mathcal{C} \Re_{n-1}^{0}\right)$ is less than $\epsilon_{n}$ for every $n$ (where $\Re_{0}$ denotes the empty set).

A proof is made in the section following.

2.15. Since $t(w)$ is uniformly continuous on $\Re_{n}$ there exists a positive number $\eta_{n}$ such that the oscillation $O\left(\eta_{n}, t, \Re_{n}\right)$ is less than $\epsilon_{n} / 3$ for every positive integer $n$ (see 1.17). Let $\mathcal{T}_{1}$ be any rectilinear triangulation of $\Re_{1}$ such that every triangle $\delta_{1}$ in $\mathcal{V}_{1}$ has diameter less than $\eta_{1}$ and those triangles $\delta_{1}$ in $\mathcal{\sigma}_{1}$ which meet the boundary $\Re_{1} \mathcal{C} \Re_{1}^{0}$ have diameters less than $\eta_{2}$; denote the vertices of $\mathcal{T}_{1}$ generically by $w_{1}$, their number by $m_{1}$. Associate with these $m_{1}$ vertices $w_{1}$, in a biunique fashion, $m_{1}$ distinct points $z_{1}$ in the $z$-plane, no three of which are collinear, such that $\left|t\left(w_{1}\right)-z_{1}\right|$ is less than $\epsilon_{1} / 9$ for corresponding points $w_{1}$, and $z_{1}$, and $\left|t\left(w_{1}\right)-z_{1}\right|$ is less than $\epsilon_{2} / 9$ for corresponding points $w_{1}$ and $z_{1}$ when $w_{1}$ is in $\Re_{1} \mathcal{C} \Re_{1}^{0}$. Put $t_{1}\left(w_{1}\right)$ equal to $z_{1}$, for corresponding points $w_{1}$, and $z_{1}$, and let $t_{1}(w)$ be the uniquely determined affine transformation on every $\delta_{1}$ in $\mathcal{G}_{1}$. It is readily verified that the transformation $T_{1}: z=t_{1}(w), w \in \Re_{1}$, is continuous and polyhedral, and the distance $\rho\left(T_{1}, T, \Re_{1}\right)$ is less than $\epsilon_{1}$. Assume that $\mathcal{T}_{n-1}$ and $T_{n-1}$ are defined. Let $\mathcal{T}_{n}$ be a rectilinear triangulation of $\Re_{n}$ such that every triangle $\delta_{n-1}$ in $\mathcal{T}_{n-1}$ also belongs to $\mathcal{T}_{n}$, every triangle $\delta_{n}$ in $\mathcal{T}_{n}$ but not in $\mathcal{T}_{n-1}$ has diameter less than $\eta_{n}$, and those triangles $\delta_{n}$ of $\mathcal{T}_{n}$ which meet the boundary $\Re_{n} \mathcal{C} \Re_{n-1}^{0}$ have diameters less than $\eta_{n+1}$; denote the vertices of $\mathcal{G}_{n}$ generically by $w_{n}$, their number by $m_{n}$. Observe that every vertex of $\mathcal{G}_{n-1}$ is also a vertex of $\mathcal{G}_{n}$; consequently $m_{n}$ exceeds $m_{n-1}$. Associate with these $m_{n}$ vertices $w_{n}$ in a biunique fashion $m_{n}$ distinct points $z_{n}$ in the $z$-plane, no three of which are collinear, such that the $m_{n-1}$ points $z_{n}$ associated with the $m_{n-1}$ vertices $w_{n-1}$ of $\mathcal{G}_{n-1}$ are the points $z_{n-1}$ assigned in the process of defining $T_{n-1},\left|t\left(w_{n}\right)-z_{n}\right|$ is less than $\epsilon_{n} / 9$ for corresponding points $w_{n}$ and $z_{n}$ when $w_{n}$ is not a vertex in $\Re_{n-1}^{0}$, and $\left|t\left(w_{n}\right)-z_{n}\right|$ is less than $\epsilon_{n+1} / 9$ for corre- 
sponding points $w_{n}$ and $z_{n}$ when $w_{n}$ belongs to $\Re_{n} \mathcal{C} \Re_{n-1}^{0}$. Put $t_{n}\left(w_{n}^{0}\right)$ equal to $z_{n}$ for corresponding points $w_{n}$ and $z_{n}$, and let $t_{n}(w)$ be the uniquely determined affine transformation on every $\delta_{n}$ in $\mathcal{T}_{n}$. It is easily verified that the transformation $T_{n}: z=t_{n}(w), w \in \Re_{n}$, is continuous and polyhedral, $T_{n}$ is identical with $T_{n-1}$ on $\Re_{n-1}$, and the distance $\rho\left(T_{n}, T, \Re_{n} \mathcal{C} \Re_{n-1}^{0}\right)$ is less than $\epsilon_{n}$. By induction, an infinite sequence of continuous polyhedral transformations $T_{n}$ is thus defined. For every $w$ in $\mathscr{D}$ there is a first $n$ such that $w$ is in $\Re_{n}$; define $t_{*}(w)$ to be $t_{n}(w)$. It is easily verified that the transformation $T_{*}: z=t_{*}(w)$, $w \in \mathcal{D}$, has the following properties: (i) $T_{*}$ is a bounded continuous transfor-

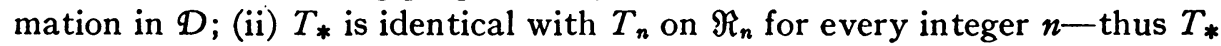
is quasi-polyhedral in $\mathcal{D}$ (see 2.13); (iii) the distance $\rho\left(T_{*}, T, \Re_{n} \mathcal{C} \Re_{n-1}^{0}\right)$ is less than $\epsilon_{n}$ for every $n$.

2.16. Return to the approximation lemma in 2.14 and its proof in 2.15 . Let $z$ be an arbitrary point in the $z$-plane. It is clear that the points $z_{n}$ chosen to correspond to the vertices $w_{n}$ of $\mathcal{G}_{n}$ may always be chosen distinct from $z$ and such that no two of them determine a line containing $z$. Then any model of $z$ under $T_{n}$ in $\Re_{n}$ must lie in the interior of some triangle $\delta_{n}$ in $\mathcal{\sigma}_{n}$. In particular, therefore, $z$ can have no models under $T_{*}$ in the boundary $\Re_{n} \mathcal{C} \Re_{n}^{0}$ for any value of $n$-that is, $T_{*}^{-1}(z) \cdot \sum_{n} \Re_{n} \mathcal{C} \Re_{n}^{0}$ is empty (see 1.2).

\section{Chapter III. A first modification}

3.1. Let $\mathcal{D}$ be any bounded domain in the $w$-plane. Suppose $T: z=t(w)$, $w \in \mathcal{D}$, is a bounded continuous transformation (see 1.2). If $z$ be any point in the $z$-plane, then $T^{-1}(z)$ is a (possibly empty) set which is closed relative to $\mathcal{D}$ (see $\left.\mathrm{R}^{2}[1,1.16]\right)$; its components $\sigma=\sigma(z, T)$ are thus of two possible types: (i) $\sigma$ may be a continuum-a closed connected set in $\mathcal{D}$-in which case it may consist of a single point in $\mathcal{D}$; (ii) $\sigma$ may not be a continuum-in which case it is a nondegenerate connected set which is closed relative to $\mathcal{D}$, but whose closure $C \sigma$ contains a point of $\mathcal{C D}$ (see 1.17). This chapter is concerned solely with components $\sigma$ of the first type; those of the second type are considered in the remaining chapters.

3.2. Given $T: z=t(w), w \in \mathcal{D}$, a bounded continuous transformation as in 3.1 , assume $z$ is a point of the $z$-plane for which $T^{-1}(z)$ has a component $\sigma=\sigma(z, T)$ which is a continuum. Let $\tau_{n}$ be any monotone decreasing sequence of positive numbers converging to zero, and consider the sets $G_{n}=G\left(\tau_{n}, z, T, \mathcal{D}\right)$ of all points $w$ in $\mathscr{D}$ for which $|t(w)-z|$ is less than $\tau_{n}$ where $n$ is any positive integer (see $\mathrm{R}^{2}[1,2.12]$ ). Clearly each $G_{n}$ is an open set containing $T^{-1}(z)$; the components of each $G_{n}$ are thus mutually exclusive connected open sets. Let $g_{n}$ denote that unique component of $G_{n}$ which contains $\sigma$ (see Newman $[1$, IV] $)$. Denote by $\pi$ the common part of the closures $C g_{n}$ of these $g_{n}$ for all positive integers $n$. Clearly $\pi \mathscr{D}$ contains $\sigma$, and $\pi \mathscr{D}$ is a subset of $T^{-1}(z)$; since $\sigma$ is a component of $T^{-1}(z)$, it follows that $\sigma$ is also a component of $\pi \mathscr{D}$. Now the $C g_{n}$ are a descending sequence of continua, so $\pi$ must be a continuum 
(see Newman $[1, \mathrm{IV}$, p. 73]). Since $\sigma$ is a component of $\pi \mathcal{D}$ whose closure $C \sigma$ is contained in $\mathcal{D}$, it follows that $\pi$ must be a subset of $\mathscr{D}$ (see Moore $[1, \mathrm{I}$, p. 23]), and hence $\pi$ is identical with $\sigma$.

3.3. Let $\Re$ be any finitely connected Jordan region in $\mathcal{D}$ containing $\sigma$ in its interior $\Re^{0}$. It follows easily that there exists a positive integer $n(\Re)$ such that $C g_{n}$ is contained in the interior $\Re^{0}$ of $\Re$ for every integer $n>n(\Re)$.

3.4. Let $T: z=t(w), w \in \mathcal{D}$, be a bounded continuous transformation as in 3.1. Assume $z$ is a point of the $z$-plane for which $T^{-1}(z)$ has a component $\sigma=\sigma(z, T)$ which is a continuum. Given an arbitrary positive number $\epsilon$, in view of 3.3 it follows that for all $n$ sufficiently large the component $g_{n}$ of $G_{n}$ which contains $\sigma$ is such that its closure $C g_{n}$ is contained in $\mathcal{D}$. Fix $n$ so that $C g_{n}$ is a subset of $\mathcal{D}$ and $\tau_{n}$ does not exceed $\epsilon / 3$; denote $g_{n}$ by $g$ and $\tau_{n}$ by $\tau$. It is readily seen that $|t(w)-z|$ has the positive value $\tau$ for $w$ in $C g C g$ (see $\mathrm{R}^{2}$ $[1,2.12])$. Let $F$ denote the set of all points $w$ in $g$ for which $|t(w)-z|$ does not exceed $\tau / 2$. Clearly $F$ is a closed subset of $g$. Consequently there exists a finitely connected oriented polygonal region $\Re$ in $g$ such that $F$ is contained in the interior $\Re^{0}$ of $\Re$ (see 2.12). Thus $|t(w)-z|$ exceeds $\tau / 2$, but is less than $\tau$ in $g C \Re^{0}$. Also the set $T^{-1}(z) \cdot g$ is contained in $\Re^{0}$. Now let $T_{*}: z=t_{*}(w)$, $w \in \Re$, be any continuous transformation such that the distance $\rho\left(T_{*}, T, \Re\right)$ (see $\mathrm{R}^{2}[1,1.9]$ ) is less than $\tau / 4$ and the number $N\left(\mathfrak{z}, T_{*}, \Re\right.$ ) is finite (cf. 2.13-2.15). One readily shows that $M\left(t_{*}-z, \Re\right)$ is less than $5 \tau / 4$ and $m\left(t_{*}-z, \Re \bigodot \Re^{0}\right)$ exceeds $\tau / 4$. Consequently $N\left(z, T_{*}, \Re\right)$ equals $N\left(z, T_{*}, \Re^{0}\right)$ that is, the models of $z$ under $T_{*}$ in $\Re$ are finite in number and lie in the interior $\Re^{0}$ of $\Re$. Thus there exists a simply-connected polygonal region $\mathfrak{r}$ in $\Re^{0}$ containing all of the models of $z$ under $T_{*}$ in its interior $\mathfrak{r}^{0}$. Since $\Re C \mathfrak{r}^{0}$ is a polygonal region on which $t_{*}(w)$ does not assume the value $z$ it follows that $\mu\left(z, T_{*}, \Re\right)$ and $\mu\left(z, T_{*}, \mathfrak{r}\right)$ are equal (see $\mathrm{R}^{2}[1,2.7$, Lemma 3$\left.]\right)$. Because $\rho\left(T_{*}, T, \Re C \Re^{0}\right)$ is less than $m\left(t_{*}-z, \Re \bigodot \Re^{0}\right)$, it follows that $\mu(z, T, \Re)$ and $\mu\left(z, T_{*}, \Re\right)$ are equal (see $\mathrm{R}^{2}[1,2.7$, Lemma 2$\left.]\right)$. Consequently $\mu(z, T, \Re)$ equals $\mu\left(\mathfrak{z}, T_{*}, \mathfrak{r}\right)$. Two cases are to be considered: the case where $\mu(\mathfrak{z}, T, \Re)$ is not zero, which is handled in 3.6 , and the case where $\mu(z, T, \Re)$ is zero, which is treated in 3.7.

3.5. The following general lemma is needed at several points in the sequel. Let there be given: (i) a simply connected Jordan region $\mathfrak{r}$ in the $w$-plane; (ii) any point $\mathfrak{w}$ contained in the interior $\mathfrak{r}^{0}$ of $\mathfrak{r}$; (iii) a single-valued, finitevalued, complex-valued continuous function $f(w), w \in \mathfrak{r C r}^{0}$; (iv) a point $z$ in the $z$-plane such that $m\left(f-\mathfrak{z}, \mathfrak{r} C \mathfrak{r}^{0}\right)$ exceeds zero. Then there exists a function $F(w), w \in \mathfrak{r}$, satisfying the following conditions: $(\alpha) F$ is defined and continuous on $\mathfrak{r} ;(\beta) F$ is identical with $f$ on $\mathfrak{r C r}^{0} ;(\gamma) F$ is different from $z$ on $\mathfrak{r} C \mathfrak{m}$, and $F(\mathfrak{w})$ is $z ;(\delta) M(F-\mathfrak{z}, \mathfrak{r})$ is equal to $M\left(f-\mathfrak{z}, \mathfrak{r} C \mathfrak{r}^{0}\right)$ (cf. $\mathrm{R}^{2}[1,2.13]$ );

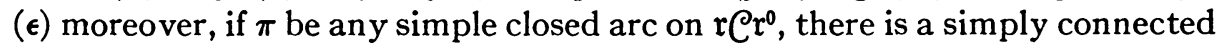
Jordan region $\mathfrak{r}(\pi)$ contained in $\mathfrak{r}$ and containing $\pi$ and $\mathfrak{w}$ on its boundary $\mathfrak{r}(\pi) \mathcal{C} \mathfrak{r}^{0}(\pi)$ such that $M[F-\mathfrak{z}, \mathfrak{r}(\pi)]$ is equal to $M(f-\mathfrak{z}, \pi)$-in fact, the values 
of $F$ in $\mathfrak{r}(\pi)$ are completely determined by the values of $f$ on $\pi$. To prove this, first assume that $\mathfrak{r}$ is the closed unit disc with center at the origin in the $w$-plane, and $\mathfrak{w}$ is the origin. Define

$$
F(w)=\left\{\begin{array}{lr}
f(w), & w \in \mathfrak{r} C \mathfrak{r}^{0} ; \\
z, & w=\mathfrak{w} ; \\
{\left[f\left(w_{*}\right)-z\right]|w|+z,} & w \in \mathfrak{r}^{0} \mathcal{C} \mathfrak{w} ;
\end{array}\right.
$$

where $w_{*}$ is the unique point of $\mathfrak{r} \mathcal{C r}^{0}$ on the ray from $\mathfrak{w}$ through $w$. It is easily verified that $F(w), w \in \mathfrak{r}$, has the desired properties. In this case, the Jordan region $\mathfrak{r}(\pi)$ may be chosen as the sector of the disc subtending the simple closed $\operatorname{arc} \pi$. Next, let $\mathfrak{r}$ be any simply connected Jordan region and $\mathfrak{w}$ be any point in $\mathfrak{r}^{0}$. There exists a topological transformation $\omega=h(w), w \in \mathfrak{r}$, mapping $\mathfrak{r}$ onto the closed unit disc $b$ with center at the origin in the $\omega$-plane, such that $0=h(\mathfrak{w})$ (see Newman $[1, \mathrm{VI}]$ ). Now this transformation maps $\mathfrak{r} \mathcal{C} \mathfrak{r}^{0}$ topologically onto $\mathfrak{d} \mathfrak{D}^{0}$. Define $g(\omega)=f h^{-1}(\omega), \omega \in \mathfrak{D C} \delta^{0}$. By what precedes, there exists a function $G(\omega), \omega \in \mathcal{D}$ satisfying conditions $(\alpha)-(\epsilon)$ with respect to $g(\omega), \omega \in \mathcal{D} \mathcal{D}^{0}$, and $z$. Define $F(w)=G h(w)$, wer. It is easily verified that $F(w), w \in \mathfrak{r}$, has the desired properties. For later use, an immediate corollary is stated. Let there be given: (i) a bounded domain $\mathcal{D}$ in the w-plane, and a simply connected Jordan region $\mathfrak{r}$ contained in $\mathcal{D}$; (ii) any point $\mathfrak{w}$ contained in the interior $\mathfrak{r}^{0}$ of $\mathfrak{r}$; (iii) a bounded continuous transformation $T: z=t(w), w \in \mathcal{D}$; (iv) a point $z$ in the $z$-plane such that $m\left(t-z, \mathfrak{r} \odot \mathfrak{r}^{0}\right)$ exceeds zero. Then there exists a transformation $T^{*}: z=t^{*}(w)$, $w \in \mathcal{D}$, satisfying the following conditions: $(\alpha) T^{*}$ is a bounded continuous transformation; $(\beta) T^{*}$ is identical with $T$ on $\mathcal{D C} \mathfrak{r}^{0} ;(\gamma) z$ has exactly one model under $T^{*}$ in $\mathfrak{r}$-namely, $\mathfrak{w}$ in $\mathfrak{r}^{0}$; $(\delta) \rho\left(T^{*}, T, \mathcal{D}\right)$ does not exceed $2 M(t-\mathfrak{z}, \mathfrak{r})$. $(\epsilon)$ if $\pi$ be any simple closed arc on $\mathfrak{r} \mathcal{C} \mathfrak{r}^{0}$, there is a simply-connected Jordan region $\mathfrak{r}(\pi)$ contained in $\mathfrak{r}$ and containing $\pi$ and $\mathfrak{w}$ on its boundary $\mathfrak{r}(\pi) \mathcal{C}^{0}(\pi)$ such that $M\left[t^{*}-\mathfrak{z}, \mathfrak{r}(\pi)\right]$ is equal to $M(t-\mathfrak{z}, \pi)$. Clearly the domain $\mathcal{D}$ may be replaced by a finitely-connected Jordan region $\Re$ containing $\mathfrak{r}$.

3.6. Assume that $\mu(\mathfrak{z}, T, \Re)$ is not zero (see 3.4). Then it follows that $\Re$ is an indicator region $(z, T)$ (see $\mathrm{R}^{2}[1,1.15]$ ), and consequently $\Re$ must contain in its interior at least one essential maximal model continuum of $z$ under $T$ in $\mathcal{D}$ (see $\mathrm{R}^{2}[1,1.16,2.16]$ ) - that is, $\kappa(z, T, \Re)$ is greater than zero (see $\left.\mathrm{R}^{2}[1,2.6]\right)$. Now let $\mathfrak{w}$ be any point in $\mathfrak{r}^{0}$. The hypotheses of the corollary in 3.5 are clearly fulfilled by $\mathfrak{r}, \mathfrak{w}, T_{*}: z=t_{*}(w), w \in \Re, \mathfrak{z}$. It is readily verified that the transformation $T^{*}: z=t^{*}(w), w \in \Re$, guaranteed by that corollary has the following properties: (i) $T^{*}$ is a continuous transformation; (ii) $T^{*}$ is identical with $T_{*}$ on $\Re \mathcal{C} \mathfrak{r}^{0}$; (iii) $z$ has exactly one model $\mathfrak{w}$ under $T^{*}$, and $\mathfrak{w}$ lies in $\Re^{0}$ - that is, $N\left(z, T^{*}, \Re\right)=N\left(\mathfrak{z}, T^{*}, \Re^{0}\right)=1$; (iv) $\mathfrak{w}$ constitutes an essential maximal model continuum for $z$ under $T^{*}$ in $\Re$-that is, $k\left(z, T^{*}, \Re\right)=1$ and $\mathfrak{w}$ belongs to $\mathcal{N}\left(T^{*}, \Re^{0}\right)$ (see $\mathrm{R}^{2}[1,1.18]$ ); (v) $M\left(t^{*}-t_{*}, \mathfrak{R}\right.$ ) is less than 
$5 \tau / 2$; (vi) $M\left(t-t^{*}, \Re \bigodot \Re^{0}\right)$ is less than $m\left(t^{*}-z, \Re C \Re^{0}\right)$. Since $\Re$ is an oriented polygonal region in $g$ such that $T\left(g \bigodot \Re^{0}\right)$ does not contain $z$ (see 3.4), it follows by 2.11 that there exists a weld $T_{f}$ of $T^{*}$ in $\Re$ to $T$ outside of $g$ with a tolerance of $\tau / 2$ and the exclusion of the value $z$ in $T_{*}\left(g \subset \Re^{0}\right)$. The properties of $T_{*}$ are summarized: $(\alpha) T_{f}$ is a bounded continuous transformation in $\mathcal{D} ;(\beta) T_{*}$ is identical with $T$ outside of $g$; $(\gamma) z$ has exactly one model $\mathfrak{w}$ under $T_{\boldsymbol{t}}$ in $g$ that is, $N\left(\mathfrak{z}, T_{f}, g\right)=1 ;(\delta) \mathfrak{w}$ constitutes an essential maximal model continuum for $z$ under $T_{f}$ in $g$ - that is, $\kappa\left(z, T_{\sharp}, g\right)=1$-and $w$ belongs to $\mathcal{N}\left(T_{f}, \mathcal{D}\right)$; ( $\epsilon) \rho\left(T_{f}, T, \mathcal{D}\right)$ is less than $\epsilon$.

3.7. Now assume that $\mu(z, T, \Re)$ is zero (see 3.4$)$; hence $\mu\left(z, T_{*}, \mathfrak{r}\right)$ is zero. Since $m\left(t_{*}-\mathfrak{z}, \mathfrak{r} C \mathfrak{r}^{0}\right)$ is positive and $\mathfrak{r}$ is simply-connected, there exists a function $f(w), w \in \Re$, having the following properties (see $\mathrm{R}^{2}[1,2.7$, Lemma 4$]$ ): (i) $f$ is defined and continuous on $\mathfrak{r}$; (ii) $f$ is identical with $t_{*}$ on $\mathfrak{r} \mathfrak{C r}^{0}$; (iii) $M(f-\mathfrak{z}, \mathfrak{r})$ equals $M\left(t_{*}-\mathfrak{z}, \mathfrak{r} C \mathfrak{r}^{0}\right)$, which is less than $5 \tau / 4$; (iv) $f$ does not take on the value $z$ in $r$. Define

$$
t^{*}(w)=\left\{\begin{array}{lr}
f(w), & w \in \mathfrak{r} ; \\
t_{*}(w), & w \in \Re \mathcal{R} .
\end{array}\right.
$$

It is easily verified that the transformation $T^{*}: z=t^{*}(w)$, $w \in \Re$, has the following properties: (i) $T^{*}$ is a continuous transformation; (ii) $T^{*}$ is identical with $T_{*}$ on $\Re C^{0}$; (iii) $z$ has no models under $T^{*}$ in $\Re$-that is, $\mathcal{N}\left(z, T^{*}, \Re\right)=0$; (iv) consequently $\kappa\left(z, T^{*}, \Re\right)=0$; (v) $M\left(t^{*}-t_{*}, \Re\right)$ is less than $5 \tau / 2$; (vi)

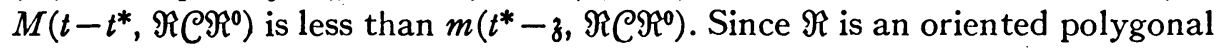
region in $g$ such that $T\left(g C \Re^{0}\right)$ does not contain $z$ (see 3.4), it follows by 2.11 that there exists a weld $T_{f}$ of $T^{*}$ in $\Re$ to $T$ outside of $g$ with a tolerance of $\tau / 2$ and the exclusion of the value $z$ in $T_{f}\left(g C \Re^{0}\right)$. The properties of $T_{t}$ are summarized: $(\alpha) T_{t}$ is a bounded continuous transformation in $\mathcal{D} ;(\beta) T_{t}$ is identical with $T$ outside of $g$; $(\gamma) z$ has no models under $T$ in $g$-that is, $N\left(z, T_{f}, g\right)=0 ;(\delta)$ consequently $\kappa\left(z, T_{\sharp}, g\right)=0 ;(\epsilon) \rho\left(T_{\sharp}, T, \mathcal{D}\right)$ is less than $\epsilon$.

3.8. The results in $\S \S 3.2-3.7$ may be described as follows.

Lemma. Let $D$ be a bounded domain in the w-plane. Given a bounded continuous transformation $T: z=t(w), w \in \mathcal{D}$, and a positive number $\epsilon$, if $z$ is any point in the z-plane whose inverse set $T^{-1}(z)$ has a component $\sigma$ which is a continuum, there is a domain $g$ containing $\sigma$ whose closure $\mathcal{C g}$ is contained in $\mathcal{D}$, whose image under $T$ lies in a $\tau$-neighborhood of $z$, where $\tau$ is a positive constant less than $\epsilon / 3$, whose frontier $\mathrm{CgCg}$ is mapped by $T$ into a subset of the circle with center $z$ and radius $\tau$. It is possible to modify $T$ in $\mathrm{g}$, moving the image of each point less than $\epsilon$, so that continuity is retained and $z$ has at most one model under the modified transformation $T_{\text {f }}$ in $g$. If $z$ has a model under $T_{t}$ in $g$, then $z$ has at least one essential maximal model continuum under $T$ in $g$; if $z$ has exactly one essential maximal model continuum under $T$ in $g$, then $z$ has a model under $T_{\mathrm{f}}$ in $\mathrm{g}$. 
3.9. Let $T: z=t(w), w \in \mathcal{D}$, be a bounded continuous transformation as in 3.1 , and suppose that $\Re$ is any finitely connected polygonal region in $\mathscr{D}$ (see 2.12). If $\tau$ be an arbitrary positive number, there exists a positive number $\delta=\delta(\tau, T, \Re)$ such that, if $w^{\prime}$ and $w^{\prime \prime}$ are any two points in $\Re$ whose distance does not exceed $\delta$, then the distance between their images $t\left(w^{\prime}\right)$ and $t\left(w^{\prime \prime}\right)$ is less than $\tau$. It may be assumed without loss of generality that $\delta$ is chosen so that to every point $w$ in $\Re$ there corresponds a linear segment contained in $\Re$ with length $\delta$ and an end point at $w$. Let $z$ be any point in the $z$-plane. Suppose that $g$ is any component of $G=G(\tau, z, T, \mathcal{D})$ (see 3.2) which contains a point of $T^{-1}(z) \Re$. It follows at once that the area of $g$ must exceed a positive constant $\zeta(\delta, \Re)$. Since $\Re$ is bounded and the components $g$ of $G$ are mutually exclusive, the number of components $g$ which contain a point of $T^{-1}(z) \Re$ must be finite.

3.10. Again, let $T: z=t(w)$, $w \in \mathcal{D}$, be a bounded continuous transformation, let $z$ be any point in the $z$-plane, and let $\epsilon$ be an arbitrary positive number. Choose any sequence of finitely connected polygonal regions $\Re_{n}$ such that $\Re_{n}$ is contained in $\Re_{n+1}^{0}$ for every $n$ and the point set sum of the $\Re_{n}$ is identical with $\mathcal{D}$ (see 2.12). It follows that if $F$ is any closed set in $\mathcal{D}$ there is a positive integer $n(F)$ such that $F$ is contained in $\Re_{n}^{0}$ for every $n$ exceeding $n(F)$. Set $\tau_{n}$ equal to $\epsilon /(3 n)$ for every positive integer $n$, and denote $G\left(\tau_{n}, z, T, \Phi\right)$ by $G_{n}$ (see 3.2). Put $\delta_{0}$ equal to 1 , and $\delta_{n}$ equal to the smaller of $\delta_{n-1}$ and $\delta\left(\tau_{n}, T, \Re_{n}\right)$ (see 3.9). Clearly $\delta_{n}$ is a monotone nonincreasing sequence of positive numbers. Let $K_{1}$ be the class of all components $g$ of $G_{1}$ such that the closure $C g$ is contained in $\mathcal{D}$ and the area of $g$ exceeds $\zeta\left(\delta_{1}, \Re_{1}\right)$; denote by $K_{1}$ the point set sum of the elements $g$ in $K_{1}$. Since $\mathcal{D}$ is bounded and the sets in $K_{1}$ are mutually exclusive and have areas exceeding a fixed positive constant, it follows that $K_{1}$ is a finite class. Moreover, for each $g$ in $K_{1}$, the function $|t(w)-z|$ has the value $\tau_{1}$ on $C g C g$ (see 3.4). In each element $g$ of $K_{1}$ which contains a point of $T^{-1}(z)$ modify $T$ in accordance with the lemma in 3.8 to obtain a new transformation $T_{1}: z=t_{1}(w)$, weD, having the following properties: (i) $T_{1}$ is a bounded continuous transformation in $\mathscr{D}$; (ii) $T_{1}$ is identical with $T$ in $\mathscr{D C} K_{1}$; (iii) the number of models for $z$ under $T_{1}$ in $K_{1}$ is not greater than the number of essential maximal model continua for $z$ under $T$ in $K_{1}$-that is, $N\left(z, T_{1}, K_{1}\right) \leqq \kappa\left(z, T, K_{1}\right)$; (iv) for each $g$ in $K_{1}$ the set $g T_{1}^{-1}(z)$ is either empty or consists of a single point; (v) $\rho\left(T_{1}, T, \mathcal{D}\right)$ is less than $\epsilon$. Assume that $K_{n-1}, K_{n-1}, T_{n-1}$ have been defined to satisfy relations analogous to (i)-(v). Let $K_{n}$ be the class of all components $g$ of $G_{n}$ such that $C g$ is contained in $\mathscr{D}$, the area of $g$ exceeds $\zeta\left(\delta_{n}, \Re_{n}\right)$, and $g K_{n-1}$ is empty. Denote by $K_{n}$ the point set sum of $K_{n-1}$ and of all the elements $g$ in $K_{n}$. It is clear that $K_{n}$ is a finite class and $T_{n-1}$ is identical with $T$ in every $g$ in $K_{n}$. Moreover, for each $g$ in $K_{n}$, the function $\left|t_{n-1}(w)-z\right|=|t(w)-z|$ has the value $\tau_{n}$ on $C g C g$. In each element $g$ of $K_{n}$ which contains a point of $T^{-1}(z)$ modify $T_{n-1}$ in accordance with the lemma in 3.8 to obtain a new transformation 
$T_{n}: z=t_{n}(w), w \in \mathcal{D}$, having the following properties: $(\alpha) T_{n}$ is a bounded continuous transformation in $\mathcal{D} ;(\beta) T_{n}$ is identical with $T$ in $\mathcal{D C} K_{n} ;(\gamma)$ for each in teger $m$ less than $n, T_{n}$ is identical with $T_{m}$ in $K_{m} ;(\delta)$ the number of models of $z$ under $T_{n}$ in $K_{n}$ is not greater than the number of essential maximal model continua for $z$ under $T$ in $K_{n}$ - that is, $N\left(z, T_{n}, K_{n}\right) \leqq \kappa\left(z, T, K_{n}\right) ;(\lambda)$ for each $g$ in $K_{n}$ the set $g T_{n}^{-1}(z)$ is either empty or consists of a single point; $(\mu) \rho\left(T_{n}, T, \mathcal{D}\right)$ is less than $\epsilon ;(\nu) \rho\left(T_{n}, T, K_{n} \subset K_{n-1}\right)$ is less than $\epsilon / n$.

3.11. Let $K$ be the point set sum of the $K_{n}$ for $n$ a positive integer; observe that the $K_{n}$ form an ascending sequence of open sets in $D$. It is clear that the continuous functions $t_{n}(w), w \in \mathcal{D}$, converge uniformly in $\mathcal{D}$ to a continuous function $t_{f}(w), w \in \mathcal{D}$. It is easily verified that the transformation $T_{f}: z=t_{f}(w), w \in \mathcal{D}$, has the following properties: $(\alpha) T_{\sharp}$ is a bounded continuous transformation in $\mathcal{D} ;(\beta) T_{\text {f }}$ is identical with $T$ in $\mathscr{D C} K ;(\gamma)$ for each positive integer $n, T_{f}$ is identical with $T_{n}$ in $K_{n} ;(\delta)$ the number of models of $z$ under $T_{t}$ in $K$ is not greater than the number of essential maximal model continua for $z$ under $T$ in $K$ - that is, $N(z, T, K) \leqq \kappa(z, T, K) \leqq \kappa(z, T, D) ;(\lambda)$ the set $T_{f}^{-1}(z) K$ is an isolated set; $(\mu) \rho\left(T_{f}, T, \mathcal{D}\right)$ is less than $\epsilon$; $(\nu)$ if $\sigma$ is any component of $T^{-1}(z)$ which is not a continuum (see 3.1), then $\sigma$ is also a component of $T_{t}^{-1}(z)$. For the component $g$ of $G_{n}$ which contains $\sigma$ is such that $C g$ must contain a point of $\mathcal{C D}$ for every $n$, and thus $\sigma$ is a subset of $\mathscr{D} \mathcal{C K}$ where $T$ is identical with $T_{\boldsymbol{f}}(\omega)$ any component $\sigma_{\boldsymbol{f}}$ of $T_{\boldsymbol{f}}^{-1}(\mathrm{z})$ either consists of a single point in $K$ or is a component of $T^{-1}(z)$ which is not a continuum (see 3.1). For if $\sigma_{f}$ has a point in common with $K$, it follows that $\sigma_{f}$ must reduce to a single point in $K$ since $T_{f}^{-1}(z) K$ is an isolated set. So suppose that $\sigma_{f}$ has no point in $K$; then $\sigma_{f}$ must also be a component of $T^{-1}(z)$ since $T_{f}$ is identical with $T$ on $\mathscr{D C K}$ and no component of $T^{-1}(z)$ may have points in both $K$ and $\mathcal{C} K$. It remains to show that $\sigma_{*}$ is not a continuum in this case. Indeed, suppose that $\sigma_{f}$ were a continuum; since it would be a closed set in $\mathcal{D}$ there must exist an $m$ such that $\sigma_{f}$ is contained in $\Re_{m}^{0}$ (see 3.10). Next, there must exist an $n$ not less than $m$ such that the component $g$ of $G_{n}$ which contains $\sigma_{f}$ is such that $C g$ is contained in $\Re_{m}$, and hence in $\Re_{n}$ (see 3.3). Since $\sigma_{H} K$ is empty, it would follow that $\sigma_{+} K_{n-1}$ is empty. And since $g$ is contained in $\Re_{n}$ it would follow that its area should exceed $\zeta\left(\delta_{n}, \Re_{n}\right)$ (see 3.9). But then $g$ should be in $K_{n}$, which is a subset of $K$, and a contradiction is obtained. So $\sigma_{f}$ is not a continuum, and its closure $C \sigma_{f}$ contains a point of $\mathcal{C D}$ (see 3.1).

3.12. Assume that $\kappa(z, T, \mathcal{D})$ is finite. Let $L$ denote the class of all the essential maximal model continua $\sigma$ for $z$ under $T$ in $\mathscr{D}$, and let $\mathcal{L}$ denote the point set sum of the $\sigma$ in $L$. Then $L$ is a finite set (see $\mathrm{R}^{2}[1,1.17]$ ), and consequently $\mathcal{L}$ is a closed set. Thus the polygonal regions $\Re_{n}$ of 3.10 may be chosen so that $\mathcal{L}$ is contained in $\Re_{1}^{0}$, and the value of $\tau_{1}$ may be required to be so small that no component $g$ in $K_{1}$ contains more than one element of $L$, and every element of $L$ belongs to a component $g$ in $K_{1}$ (see 3.3). Then the preceding construction yields $N\left(z, T_{f}, K\right)=\kappa(z, T, \mathcal{D})$ (see 3.8). This fact 
may also be established by using the lower semi-continuity properties of $\kappa(z, T, \Phi)$ (see $\mathrm{R}^{2}[1,2.18]$; also see 5.5 ).

3.13. The results obtained in $\$ \S 3.9-3.12$ may be summarized as follows.

ThEOREM. Let $\Phi$ be a bounded domain in the w-plane. Given a bounded continous transformation $T: z=t(w), w \in \mathcal{D}$, let $z$ be any point in the $z$-plane and let $\epsilon$ be any positive number. There exists a bounded continuous transformation $T_{f}: z=t_{f}(w), w \in \mathcal{D}$, such that the distance $\rho\left(T_{f}, T, \mathscr{D}\right)$ is less than $\epsilon$, and every component of $T_{f}^{-1}(z)$ either reduces to a single point which is isolated in the set $T_{\bar{f}}^{-1}(z)$ or else is a component of $T^{-1}(\mathfrak{z})$ which is not a continuum, and every component of $T^{-1}(z)$ which is not a continuum is also a component of $T_{+}^{-1}(z)$-in other words, the components of $T^{-1}(z)$ which are not continua are identical with the components of $T_{t}^{-1}(z)$ which do not reduce to single points. Moreover, the number of components of $T_{t}^{-1}(z)$ which reduce to single points does not exceed the number of essential maximal model continua for $z$ under $T$ in $D$; in case this latter number is finite, the transformation $T_{\text {t }}$ may be chosen so that the number of components of $T_{f}^{-1}(z)$ which reduce to single points is equal to the number of essential maximal model continua for $z$ under $T$ in $D$.

Thus if every component of $T^{-1}(z)$ is a continuum, the question raised in 1.10 is now answered.

\section{Chapter IV. A second modification}

4.1. Let $\mathcal{D}$ be any bounded domain in the $w$-plane. Suppose $T: z=t(w)$, $w \in \mathcal{D}$, is a bounded continuous transformation (see 1.2). Since the result to be established in this chapter is trivial if $t(w)$ is constant on $D$ (see 1.15, $4.65)$, attention is restricted in the sequel to the case when $t(w)$ is not constant on $\mathscr{D}$. Assume $z$ is a point of the z-plane for which $T^{-1}(z)$ is not empty, and no component $\sigma=\sigma(z, T)$ of $T^{-1}(z)$ is a continuum (see 3.1); hence $C \sigma$ contains a point of $\mathcal{C D}$. Since $\sigma$ is closed relative to $T^{-1}(z)$, and $T^{-1}(z)$ is closed relative to $\mathscr{D}$, it follows that $\sigma$ is closed relative to $\mathcal{D}$ - that is, $C \sigma \mathscr{D}$ reduces to $\sigma$.

4.2. Let $\tau$ be a fixed positive number, and consider the set $G=G(\tau, z, T, \mathcal{D})$ of all points $w$ in $\mathscr{D}$ for which $|t(w)-z|$ is less than $\tau$ (cf. 3.2). It may be assumed that $\tau$ is so small that there are points in $\mathscr{D}$ which are not in $G$ (see 4.1). Clearly $G$ is an open set containing $T^{-1}(z)$, and hence it is not empty. $G$ has an at most enumerable number of components $g$, each of which is a connected open set. Every component $\sigma$ of $T^{-1}(z)$ is contained in exactly one component $g$ of $G$; if a component $g$ of $G$ has points in common with a component $\sigma$ of $T^{-1}(z)$, then $g$ contains $\sigma$.

4.3. In the sequel it is necessary to consider various classes of point sets in $\Phi$; each shall be denoted by a capital letter $K, L$, or $M$, followed by suitable subscripts and superscripts. The point set sum of the sets in a class $K, L$, or $M$ will be denoted by the corresponding capital script letter $K, \mathcal{L}$, or $\mathcal{N}$, respectively, followed by the same subscripts and superscripts. 
4.4. Let $K_{0}$ denote the class of all components $g$ of $G$ which contain a point of $T^{-1}(z)$ (see 4.2). Evidently $K_{0}$ is not empty; $K_{0}$ contains an at most enumerable number of elements. Moreover, $K_{0}$ is an open set, whose components are the sets in $K_{0}$. In particular, if $g$ be any element of $K_{0}$ it follows that $C g K_{0}$ reduces to $g$. The set $K_{0}$ contains $T^{-1}(z)$. If $g$ be any element of $K_{0}$, then $g$ must contain a component $\sigma$ of $T^{-1}(z)$ (see 4.2), and hence $C g$ must contain a point of $\mathcal{C D}$ (see 4.1). If $g$ is any element of $K_{0}$, it is now easily verified that the following relations hold.

$$
C\left[g T^{-1}(z)\right] \Phi=C g T^{-1}(z)=g T^{-1}(z) \neq 0, \quad C\left[g T^{-1}(z)\right] \mathcal{C D} \neq 0 .
$$

4.5. Now let $\Re$ be any finitely connected oriented polygonal region in $\mathscr{D}$ (see 2.12). Denote by $K^{0}$ the class of all elements $g$ in $K_{0}$ which have points in $\Re$; thus $K_{0} \Re \subset K^{0}$. Denote by $K^{01}$ the class of all elements $g$ in $K^{0}$ for which $g \Re T^{-1}(z)$ is not empty. Clearly the sets $K^{0}, K^{01}, K^{0} \mathcal{C} K^{01}$ are open, and $\Re T^{-1}(z)$ is a subset of $K^{01}$. Of course, $K^{0}$ may be empty; for the purpose of the present investigation, this case is trivial, and hence it is excluded in the sequel. Since $K^{0}$ is a class of components of $K_{0}$, it follows that $C K^{0} \cdot K_{0}$ reduces to $K^{0}$ (see 4.4).

4.6. Suppose $g$ is an element of $K^{0}$. Let $K^{1}(g)$ denote the class of all components $g^{1}$ of the open set $g \bigodot \Re$; evidently $K^{1}(g)=g C \Re$. Let $K^{11}(g)$ denote the class of all elements $g^{1}$ in $K^{1}(g)$ for which $C\left[g^{1} T^{-1}(z)\right]$ contains a point of $\mathcal{C D}$, and let $K^{12}(g)$ denote the class of all elements $g^{1}$ in $K^{1}(g)$ and not in $K^{11}(g)$. For $i$ equal to 1 or 2 , let $K^{1 i 1}(g)$ denote the class of all elements $g^{1}$ in $K^{1 i}(g)$ for which $C g^{1}$ contains a point of $\Re T^{-1}(z)$. Clearly the sets $K^{1}(g), K^{1 i}(g)$, $K^{1 i 1}(g), K^{1 i}(g) \mathcal{C} K^{1 i 1}(g)$ are all open sets, for $i$ equal to 1 or 2 . It is quite important, but not quite clear, that the class $K^{11}(g)$ is never empty. A proof of this fact is made in 4.8 .

4.7. The following general lemma is needed in the sequel. Assume 2 and $G$ are two open sets such that the common part of their complements, ¿2CG, is empty. Let $f$ be a bounded closed connected set which contains points of $\mathcal{C} 2$ and of $\mathcal{C} G$. Then there exists a connected set $h$ in $f 2 G$ such that $C h$ contains a point of $\mathcal{C} 2$. A proof may be readily constructed from the theorems in Chapter I of Moore [1].

4.8. Let $g$ be any element of $K^{0}$. In view of 4.5 and 4.4 , it follows that there exists a component $\sigma$ of $T^{-1}(z)$ which is contained in $g$. If $\sigma$ is contained in $\mathcal{R}$, then $\sigma$ must be in some component $g^{1}$ of $g \mathcal{C}$; hence $C\left[g^{1} T^{-1}(z)\right]$ contains $C \sigma$ which has a point in $\mathcal{C D}$ by 4.1 . Thus $g^{1}$ belongs to $K^{11}(g)$. If $\sigma$ is not contained in $\varrho \Re$, then $C \sigma$ is a bounded closed connected set which contains points of $\mathcal{C D}$ and of $\Re$. By the lemma in 4.7, it follows that there is a connected set $\sigma_{*}$ of $C \sigma \mathscr{D} \mathcal{C}=\sigma \mathcal{C} \Re$ (see 4.1) such that $C \sigma_{*}$ contains a point of $\mathcal{C D}$. Applying the preceding reasoning to $\sigma_{*}$ instead of $\sigma$ shows that there exists a $g^{1}$ belonging to $K^{11}(g)$. Thus $K^{11}(g)$ is never empty (see 4.6).

4.9. Let $K^{1}$ be the class of all elements $g^{1}$ belonging to a $K^{1}(g)$ for $g$ in 
$K^{0}$ (see 4.6); evidently $K^{1}=K^{0} \bigodot \Re$. Similarly, let $K^{1 i}, K^{1 i 1}$, be the class of all elements $g^{1}$ belonging to a $K^{1 i}(g), K^{1 i 1}(g)$, respectively for $g$ in $K^{0}$, where $i$ equals 1 or 2 . The point sets $K^{1}, K^{1 i}, K^{1 i 1}, K^{1 i} C K^{1 i 1}$, are clearly open. If $g$ be any element of $K^{0}$, it is obvious that $K^{1} g, K^{1 i} g, K^{1 i 1} g$ are identical with $K^{1}(g), K^{1 i}(g), K^{1 i 1}(g)$, respectively, for $i$ equal to 1 or 2 .

4.10. Suppose $g$ is an element of $K^{0}$ for which $K^{1 i 1}(g)$ is not empty, where $i$ equals 1 or 2 . Then $K^{1 i 1}(g)$ contains an element $g^{1}$, and $C g^{1}$ contains a point of $\Re T^{-1}(z)$ (see 4.6$)$; consequently $C g \Re T^{-1}(z)$ is not empty. In view of the relations in 4.4 , it follows that $g \Re T^{-1}(z)$ is not empty, so $g$ must belong to $K^{01}$ (see 4.5). From 4.9 there follow, for $i$ equal to 1 and 2, the relations $K^{1 i 1}=\sum K^{1 i 1}(g), K^{1 i 1}=\sum K^{1 i 1} g$, the summation extending over the class $K^{01}$.

4.11. The following closure relations are needed in the sequel.

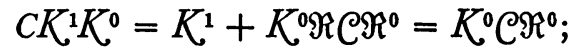

$$
\begin{aligned}
& C K^{1 i} K^{0}=K^{1 i}+C K^{1 i} K^{0} \Re C \Re^{0} \text {, } \\
& i=1,2 \text {; } \\
& C K^{1 i 1} K^{0}=K^{1 i 1}+C K^{1 i 1} K^{0} \Re \Re^{0} \text {, } \\
& i=1,2 \text {; } \\
& C K^{1} g=K^{1} g+g \Re C \Re^{0}=g C \Re^{0}, \\
& g \in K^{0} \text {; } \\
& C K^{1 i} g=K^{1 i} g+C K^{1 i} g \Re \circlearrowright \Re^{0} \text {, } \\
& i=1,2, g \in K^{0} \text {; } \\
& C K^{1 i 1} g=K^{1 i 1} g+C K^{1 i 1} g \Re C \Re^{0} \text {, } \\
& i=1,2, g \in K^{0} \text {; } \\
& C g^{1} g=g^{1}+C g^{1} g \Re \bigodot \Re^{0} \text {; } \\
& g^{1} \in K^{1} \text {. }
\end{aligned}
$$

A proof of (1) may be made as follows (see Newman [1, II, V]; 4.9).

$$
\begin{aligned}
& C K^{1} K^{0}=C\left(K^{0} \bigodot \Re\right) K^{0}=K^{0} C \bigodot \Re=K^{0} \bigodot \Re^{0} \\
& =K^{0} \bigodot \Re+K^{0} \Re \bigodot \Re^{0}=K^{1}+K^{0} \Re \bigodot \Re^{0} .
\end{aligned}
$$

The others follow easily from (1); also relation (7) may be obtained directly from the fact that $g^{1}$ is a component of $g \subset \Re$.

4.12. Using relations in $4.4,4.6,4.11$ (7), the reader may readily verify that, for every element $g^{1}$ in $K^{1}(g)$, the following equations hold (see 1.17).

$$
\text { fr } g^{1} T^{-1}(z)=C g^{1} \mathcal{C} g^{1} g \Re C \Re^{0} T^{-1}(z)=C g^{1} \Re T^{-1}(z) .
$$

4.13. If $g$ be any element of $K^{0}$, it will now be shown that $K^{12} g T^{-1}(z)$ is a subset of $K^{121} g$ (see 4.6); consequently $K^{12} T^{-1}(z)$ is a subset of $K^{121}$ (see 4.9). For if $\mathfrak{w}$ is any point of $K^{12} g T^{-1}(z)$, there exists an element $g^{1}$ of $K^{12}$, and a component $\sigma$ of $T^{-1}(z)$ such that $g^{1} \sigma$ is not empty. Since $C \sigma$ contains a point of $\mathcal{C D}$ (see 4.1), it follows that $\mathrm{Cg}^{1} \sigma$ is not empty; otherwise $C \sigma$ would be a subset of $C\left[g^{1} T^{-1}(z)\right]$ which is in turn a subset of $\mathcal{D}$ (see 4.6). Since $\sigma$ is a connected set, it is true that $\mathrm{fr}^{\mathrm{g}^{1} \sigma}$ is not empty (see Newman [1, IV]). In view of 4.12 and 4.6 , one concludes that $g^{1}$ must belong to $K^{121}(g)$, and hence $\mathfrak{w}$ is contained in $K^{121} \mathrm{~g}$.

4.14. Using $4.5,4.6,4.9$, the reader will have no difficulty in showing that 
the following relations are true.

$$
\begin{aligned}
K^{0} \mathcal{C} K^{11} & =K^{0} \Re+K^{12} \neq 0 ; \\
g C K^{11} & =g C\left(K^{11} g\right)=g \Re+K^{12} g \neq 0, \quad g \in K^{0} .
\end{aligned}
$$

4.15. Suppose $g$ is an element of $K^{0}$ (see 4.5). Let $K^{2}(g)$ denote the class of all components $h$ of $g C K^{11}$ (see 4.6,4.8); in view of 4.14 (2), it is clear that $K^{2}(g)=g C K^{11}=g \Re+K^{12} g$. Let $K^{21}(g)$ denote the class of all components $h$ in $K^{2}(g)$ which contain a point of $T^{-1}(z)$. Obviously $K^{2}(g) T^{-1}(z)$ is a subset of $K^{21}(g)$.

4.16. Let $K^{2}$ be the class of all elements $h$ belonging to a $K^{2}(g)$ for $g$ in $K^{0}$; in view of $4.14(1)$, it follows that $K^{2}=K^{0} \mathcal{C} K^{11}=K^{0} \Re+K^{12}$. Let $K^{21}$ be the class of all elements $h$ belonging to a $K^{21}(g)$ for $g$ in $K^{0}$. It is clear that $K^{2} T^{-1}(z)$ is a subset of $K^{21}$. If $g$ is any element of $K^{0}$, it follows that $K^{2} g$, $K^{21} g$ are identical with $K^{2}(g), K^{21}(g)$, respectively (cf. 4.9 ).

4.17. Suppose $g$ is an element of $K^{0}$ for which $K^{21} g$ is not empty. It follows from 4.15 that $g \Re T^{-1}(z)+K^{12} g T^{-1}(z)$ is not empty. If $g \Re T^{-1}(z)$ is not empty, then $g$ belongs to $K^{01}$ (see 4.5). But if $K^{12} g T^{-1}(z)$ is not empty, it follows from $4.13,4.6,4.12$, that $g \Re T^{-1}(z)$ is not empty either. Thus $g$ must belong to $K^{01}$. From 4.16 there follow the relations $K^{21}=\sum K^{21}(g), K^{21}=\sum K^{21} g$, the summation extending over the class $K^{01}$ (cf. 4.10).

4.18. The following general lemma is needed in the sequel. Assume 2 and $G$ are two open sets, and $h$ is a component of $2 \mathcal{C} G$. Then the interior $h^{0}$ of $h$ is identical with the set $h \subset C G$, and $h \mathrm{fr} h$ reduces to $h C G$ (see 1.17). A proof may be constructed from the theorems in Chapter IV of Newman [1].

4.19. As an immediate corollary to the general lemma in 4.18, there follow the relations $h^{0}=h C C\left(K^{11} g\right)=h C C K^{11}$ and $h$ fr $h=h C\left(K^{11} g\right)=h C K^{11}$ for every element $h$ in $K^{2}(g)$ where $g$ is in $K^{0}$ (see $4.9,4.14,4.15,4.16$ ).

4.20. Thus far, the fact that the Jordan region $\Re$ is an oriented polygonal region (see 4.5) has not been used, but it will play an important role in the sequel. Let $\delta(\Re)$ be the shortest distance between nonadjacent sides, considered as point sets, of the oriented polygons in $\Re C \Re^{0}$ - the sides entering in to this definition do not necessarily belong to the same polygon. Clearly $\delta(\Re)$ is a positive number not greater than the side length of any polygon in $\Re \mathcal{C} \Re^{0}$.

4.21. A square $s$ in the $w$-plane is said to be diagonally oriented if its diagonals are parallel to the $u$ - and $v$-axes in some order. Diagonally oriented squares have the following valuable property. Let $s_{1}$ and $s_{2}$ be two diagonally oriented open squares with centers on $\Re \mathcal{R} \Re^{0}$ and diagonal lengths not exceeding $\delta(\Re)$. If $s_{1}$ and $s_{2}$ have a point in common, then $s_{1}$ and $s_{2}$ have a point in common with $\Re C \Re^{0}$. A proof will be indicated. Denote the centers of $s_{i}$ by $w_{i}$ for $i$ equal to 1 and 2 . Since $s_{1}$ and $s_{2}$ have a point in common, $\left|w_{1}-w_{2}\right|$ must be less than $\delta(\Re)$, and consequently $w_{1}$ and $w_{2}$ cannot belong to nonadjacent sides of $\Re$-that is, either $w_{1}$ and $w_{2}$ belong to adjacent sides of an 
oriented polygon in $\Re \subset \Re^{0}$, or they belong to the same side of an oriented polygon in $\Re C \Re^{0}$. An elementary reasoning treats both of these cases to make the proof.

4.22. Now let $\mathfrak{r}$ be any finitely connected Jordan region in $\mathscr{D}$ which contains $\Re$ in its interior $\mathfrak{r}^{0}$ (see 2.12 ), and put $\delta(\Re, \mathfrak{r}$ ) equal to the distance between $\Re$ and $\mathcal{C r}^{0}$. Clearly $\delta(\Re, \mathfrak{r})$ is a positive number. If $s$ be a diagonally oriented open square having a point in $\Re$ and a diagonal length not exceeding $\delta(\Re, \mathfrak{r})$, then $s$ is contained in $\mathfrak{r}^{0}$.

4.23. Let $s$ be any diagonally oriented open square with center $\mathfrak{w}$ on $\Re C \Re^{0}$ and diagonal length not exceeding the smaller of $\delta(\Re) / 2$ and $\delta(\Re, \mathfrak{r})$. Suppose $\mathfrak{w}$ is on a side $w_{1} w_{2}$ of an oriented polygon in $\Re \mathcal{C} \Re^{0}$ (see 2.3 ); then (see 4.20) $\left|w_{1}-\mathfrak{w}\right|+\left|\mathfrak{w}-w_{2}\right|=\left|w_{1}-w_{2}\right| \geqq \delta(\Re)$, and consequently one of the points $w_{1}, w_{2}$ is not in $s$-let the notations be chosen so that $w_{2}$ is not in $s$. If $w_{2} w_{3}$ is the side of the oriented polygon in $\Re C \Re^{0}$ adjacent to $w_{1} w_{2}$ with common end point $w_{2}$, it follows that $w_{2} w_{3}$ does not meet $s$. If $w_{0} w_{1}$ is the other side of this oriented polygon adjacent to $w_{1} w_{2}$, then necessarily $w_{0} w_{1}$ is perpendicular to $w_{1} w_{2}$. An elementary reasoning shows that the set $s \Re \mathcal{C} \Re^{0}$ must be contained in $w_{0} w_{1}+w_{1} w_{2}$. From plane topology (see Newman [1]), the following facts emerge (see 4.22): $(\alpha) d_{0} \equiv s \mathcal{\cap}$ is a nonempty connected open set in $\mathfrak{r}^{0} \mathcal{R} ;(\beta) d \equiv s \Re^{0}$ is a nonempty connected open set in $\Re^{0} ;(\gamma) s \Re \bigodot \Re^{0}$ is a connected set in $C d_{0} \cdot C d ;(\delta)$ neither of the measures $\left|d_{0}\right|,|d|$ is less than $|s| / 4$ (see 1.17).

4.24. Since $t(w)$ is continuous on the bounded set $\mathcal{D}$ (see 4.1 ), it follows that for every positive number $\epsilon$ and every closed set $F$ in $\mathcal{D}$, there is a positive number $\delta(\epsilon, T, F)$ such that $O[\delta(\epsilon, T, F), t, F]$ is less than $\epsilon$ (see 1.17). Let $s$ be a diagonally oriented open square with center on $\Re T^{-1}(z)$ and diagonal length not exceeding the smaller of $\delta(\Re, \mathfrak{r})$ and $2 \delta(\tau, T, \mathfrak{r})$ (see 4.2). Then $s$ is contained in $\mathfrak{r}^{0}$ (see 4.22), and consequently $s$ is contained in $G$ (see 4.2). Since $s$ is connected, it is contained in a component $g$ of $G$, and $g$ is an element of $K^{01}$ (see 4.5 ).

4.25. In the sequel, $\delta_{0}=\delta_{0}(\tau, T, \Re, \mathfrak{r})$ will denote the smallest of the numbers $\delta(\Re) / 2, \delta(\Re, \mathfrak{r}), 2 \delta(\tau, T, \mathfrak{r}$ ) (see $4.20,4.22,4.24)$. Obviously $\delta_{0}$ is positive. Let $g$ be any element of $K^{01}$ (see 4.5). If $s$ is any diagonally oriented open square with center in $g \Re T^{-1}(\xi)$ and diagonal length not exceeding $\delta_{0}$, it follows that $s$ is contained in $\mathrm{gr}^{0}$ (see 4.24).

4.26. As an immediate corollary to the preceding remark and the fact that the elements in $K^{01}$ are mutually exclusive and contained in the bounded set $\mathcal{D}$, it follows that $K^{01}$ is a finite class.

4.27. Let $g$ be any element of $K^{0}$ (see 4.5). Pick any point $\mathfrak{w}$ in $g \Re C \Re^{0}$. In view of 4.15 it is clear that $\mathfrak{m}$ is contained in a unique element $h$ of $K^{2}(g)$. Since $g$ is open (see 4.2), there is a diagonally oriented open square $s$ with center $\mathfrak{w}$ and diagonal length not exceeding $\delta_{0}$ which is contained in $g$. In view of 4.25 , relations $(\alpha),(\beta),(\gamma),(\delta)$ of 4.23 are valid. Moreover, the reader will 
further verify that the following statements are true: $(\xi) d_{0}=s \mathcal{C}=s K^{1} g$ is contained in a unique element $g^{1}$ of $K^{1}(g)$ (see 4.6); $(\eta) s \Re$ is a connected subset of $h$ (see 4.15); ( $\zeta) d=s \Re^{0}$ is contained in $h^{0}$ (see 4.19); $(\lambda) \mathfrak{w}$ is contained in $s \Re C \Re^{0}$ which is a subset of $C g^{1} h$; $(\mu)$ if $g^{1}$ belongs to $K^{12}(g)$ then $s$ is a subset of $h^{0}$, if $g^{1}$ belongs to $K^{11}(g)$ then $s h$ is identical with $s \Re$; $(\nu)$ in any case, $s$ is a subset of $h+K^{11} g$.

4.28. Let $g$ be any element of $K^{0}$. It will now be shown that the classes $K^{1 i 1}(g)$ are finite, for $i$ equal to 1 or 2 (see 4.6). Indeed, for any $g^{1}$ in $K^{1 i 1}(g)$ it is true that $C g^{1} g \Re \bigodot \Re^{0} T^{-1}(z)$ is not empty (see $\left.4.6,4.12\right)$. Let $s$ be a diagonally oriented open square with center in the set $C g^{1} g \Re C \Re^{0} T^{-1}(z)$ and diagonal length $\delta_{0}$; then $s$ is contained in $g$ by 4.25. Since $s$ contains points of $g^{1}$, it follows from $4.27(\xi)$ that $d_{0}$ is a subset of $g^{1}$. From $4.23(\delta)$ one obtains the fact that $\left|g^{1}\right|$ is not less than $\delta_{0}^{2} / 8$. Because the elements of $K^{1 i 1}(g)$ are mutually exclusive and are contained in the bounded set $\mathscr{D}$ it follows that $K^{1 i 1}(g)$ is a finite class.

4.29. As an immediate corollary to $4.10,4.26,4.28$, it follows that the classes $K^{1 i 1}$ are finite for $i$ equal to 1 or 2 .

4.30. The following general lemma is needed. Assume $e$ is any connected set whose interior $e^{0}$ (see 1.17) is disconnected. Let $e^{0}=2_{1}+22$, where $2_{i}$ are nonempty mutually exclusive open sets, be any partition of $e^{0}$ (see Newman $[1$, IV] $)$. Then either $(\alpha) e$ contains a point of $\mathcal{C} C e^{0}$, or $(\beta) e \mathcal{C} e^{0}$ contains a point of $C 2_{1} C 2_{2}$. The proof is left for the reader.

4.31. It will now be shown that every $h$ in $K^{2}$ (see 4.16) has a connected interior $h^{0}=h C C K^{11}$ (see 4.19). Indeed, suppose there is a $g$ in $K^{0}$ for which $K^{2}(g)$ contains an $h$ whose interior $h^{0}$ admits of a partition $2_{1}+2_{2}$, where the $2_{i}$ are nonempty mutually exclusive open sets. In view of the lemma in 4.30 two possibilities may occur. ( $\alpha$ ) Suppose $\mathfrak{w}$ is a point of $h \mathcal{C} C h^{0}$. It follows from 4.15 and 4.11 that $w$ belongs to $h C K^{11}$ which is a subset of $g C K^{11} C K^{11} \Re \mathcal{C} \Re^{0}$. Since $g C C h^{0}$ is open, there exists a diagonally oriented open square $s$ with center $\mathfrak{w}$ and diagonal length not exceeding $\delta_{0}$ such that $g C C h^{0}$ contains $s$. Then $s$ does not have any points in $h^{0}$, contrary to $4.27(\zeta)$. Thus $(\alpha)$ cannot occur. ( $\beta$ ) Suppose $\mathfrak{w}$ is a point of $h C h^{\circ} C 2_{1} C 2_{2}$. As in case $(\alpha), \mathfrak{w}$ must belong to $g C K^{11} \mathcal{C} K^{11} \Re C \Re^{0}$. Let $s$ be any diagonally oriented open square in $g$ with center $\mathfrak{w}$ and diagonal length not exceeding $\delta_{0}$. From $4.23(\beta)$ and $4.27(\zeta)$ it follows that $d$ is a subset of just one of $2_{1}$ and $2_{2}$-assume notations are so chosen that $d$ is a subset of $2_{1}$. From 4.6, 4.9, 4.27, and the fact that $s$ meets $K^{11}$, it follows that $d_{0}$ must be a subset of $K^{11}$, and hence of $\mathcal{C} h$. Since $s \Re$ is a subset of $C d$, one has the relations

$$
s 2_{2}=s \Re 2_{2}+d_{02} \subset C 2_{12}+C h 2_{2}=0,
$$

which contradict the fact that $w$ belongs to $s C 2_{2}$. So $(\beta)$ cannot occur either. Thus $h^{0}$ must be connected.

4.32. Let $g$ be an element of $K^{0}$ and $h$ an element of $K^{2}(g)$ (see 4.15). 
Since $h$ is a component of $g C K^{11}$, and $K^{11}$ is open (see 4.9), it follows that $C h g$ is identical with $h$ (see Newman [1, IV]). Now $g$ is a connected set which contains $h$ and also points of $K^{11}$, which is a subset of $\mathcal{C} h$ (see 4.8); thus fr $h g$ is not empty (see Newman [1, IV]). Using the preceding relation, together with those in $4.11,4.15,4.18,4.19$, one may verify that the following relations hold.

$$
0 \neq \text { fr } h g=h C C h=h C K^{11}=h C K^{11} \Re C \Re^{0} .
$$

4.33. Again, let $g$ be an element of $K^{0}$, and $h$ an element of $K^{2}(g)$. Define $K(h)$ to be the class of all diagonally oriented open squares in $g$ having centers on $h C K^{11}$ and diagonal lengths not exceeding $\delta_{0}$ (see 4.25). In view of 4.32 this class is not empty. Using $4.21,4.23,4.25,4.27$, the reader may easily verify that the sets $K(h)$ (see 4.3 ) have the following properties: $(\alpha) K(h) \subset g x^{0}$, $h C K^{11} \subset K(h) \subset h+K^{11} g \subset g ;(\beta) K(h)$ is an open set; $(\gamma)$ if $h_{1}, h_{2}$ are two distinct elements of $K^{2}$, then

$$
K\left(h_{1}\right) h_{2}=0=K\left(h_{1}\right) K\left(h_{2}\right) .
$$

4.34. With the same assumptions as in 4.33 , define $2(h)$ to be the set $h^{0}+K(h)$ (see 1.17). It is readily verified that the sets $2(h)$ satisfy the following conditions: $(\alpha) h \subset 2(h)=h+K(h) \subset h+K^{11} g \subset g ;(\beta) 2(h)$ is a bounded connected open set; $(\gamma)$ if $h_{1}, h_{2}$ are two distinct elements of $K^{2}$, then $2\left(h_{1}\right) 2\left(h_{2}\right)=0$.

4.35. Define sets $2_{1}$ and $2_{2}$ by the relations $\sum 2(h)$, the summation extending over sets $h$ in $K^{21}$ and in $K^{2} C K^{21}$ respectively (see 4.16). Clearly $2_{1}$ and $2_{2}$ have the following properties: $(\alpha) K^{21} \subset{ }_{21} \subset K^{11}+K^{21} \subset K^{0}, K^{2} \subset K^{21}$ $C{ }_{2} \subset K^{11}+K^{2} C K^{21} \subset K^{0} ;(\beta) 2_{1}$ and $2_{2}$ are open sets; $(\gamma) 2_{1}$ and $2_{2}$ have no points in common.

4.36. The following closure relations will be useful (cf. 4.11).

$$
\begin{aligned}
C K^{2} K^{0} & =K^{2} ; \\
C K^{21} K^{0} & =K^{21} ; \\
C h g=h, & h \in K^{2}(g),
\end{aligned}
$$

$$
\begin{aligned}
C K^{2} g & =K^{2} g, & & g \in K^{0} ; \\
C K^{21} g & =K^{21} g, & & g \in K^{0} ;
\end{aligned}
$$

A proof of (1) may be made as follows (see Newman [1, II]; 4.16, 4.9).

$$
C K^{2} K^{0}=C\left(K^{0} C K^{11}\right) K^{0}=K^{0} C \mathcal{C} K^{11}=K^{0} C K^{11}=K^{2} .
$$

Statement (2) now follows from the relations

$$
C K^{21} K^{0}=C K^{21} K^{2}=K^{21}+C K^{21} K^{2} C K^{21}=K^{21},
$$

since $C K^{21} K^{2} C K^{21}$ is contained in $C 2_{12}$, which is empty (see 4.35). The others follow easily from these; also relation (5) has been verified in 4.32.

4.37. Further closure relations are expressed in these statements.

$$
C\left[K^{12} T^{-1}(z)\right] \subset C K^{121} K^{0} T^{-1}(z) ;
$$




$$
C\left[K^{12} g T^{-1}(z)\right] \subset C K^{121} g T^{-1}(z),
$$$$
g \in K^{0}
$$

Indeed, from 4.13 it follows that $K^{12} g T^{-1}(z)$ and $K^{12} T^{-1}(z)$ are identical with $K^{121} g T^{-1}(z)$ and $K^{121} T^{-1}(z)$ respectively. For an element $g^{1}$ of $K^{12}$ it is clear, in view of 4.1 and 4.4 , that $C\left[g^{1} g T^{-1}(z)\right]$ is a subset of $C g^{1} g T^{-1}(z)$. Using 4.28 and 4.29 , the reader will easily complete the proof.

4.38. Now it can be shown that the following sets are all closed.

(1) $K^{2} T^{-1}(z)$;

(2) $\quad K^{21} T^{-1}(z)$;

To prove (1), observe that 4.16 implies the identity: $K^{2} T^{-1}(z)=K^{0} \Re T^{-1}(z)$ $+K^{12} T^{-1}(z)$. By using 4.1 and 4.5 , one readily finds that $C\left[K^{0} \Re T^{-1}(z)\right]$ is a subset of $K^{2} T^{-1}(z)$; and by using 4.37 and 4.11 , one sees that $C\left[K^{12} T^{-1}(z)\right]$ is a subset of $K^{2} T^{-1}(z)$. Thus (1) is established. From 4.36 flows the identity: $K^{21} T^{-1}(z)=C K^{21} K^{2} T^{-1}(z)$. Thus (2) is demonstrated. By using 4.4 and 4.36, the reader may easily show that the remainder of the sets are closed.

4.39. For any element $h$ in $K^{2}$, the following facts will now be demonstrated (see 1.17, 4.15, 4.16).

$$
\text { fr } h T^{-1}(z)=h C \mathcal{C} h T^{-1}(z)=\left\{\begin{array}{lr}
h C K^{111} \Re \mathcal{C} \Re^{0} T^{-1}(z) \neq 0, & h \in K^{21}, \\
0, & h \in K^{2} \mathcal{C} K^{21} .
\end{array}\right.
$$

From 4.4 and 4.32 the following relations come.

$$
\text { fr } h T^{-1}(z)=h C C h T^{-1}(z)=h C K^{11} \Re C \Re^{0} T^{-1}(z) \text {. }
$$

If $h$ is not in $K^{21}$, it is clear that these sets are empty. Now assume that $h$ belongs to $K^{21}$; since it contains a point of $T^{-1}(z)$, there is a component $\sigma$ of $T^{-1}(z)$ such that $h \sigma$ is not empty. Now $C h \sigma$ cannot be empty either, for if it were, then it would follow from 4.38 (5) that $C \sigma$ must be contained in $\mathcal{D}$, contradicting 4.1. Since $\sigma$ is connected, it follows that fr $h \sigma$, and consequently fr $h T^{-1}(z)$, is not empty (see Newman [1, IV]). Let $\mathfrak{w}$ be any point of $\operatorname{fr} h T^{-1}(z)$, and consider the diagonally oriented open square $s$ with center $\mathfrak{w}$ and diagonal length $\delta_{0}$. If $h$ belongs to $K^{2}(g)$ it follows from 4.25 that $s$ is a subset of $g$. By $4.27(\xi)$, the set $s K^{1} g$ is contained in a unique element $g^{1}$, which belongs to $K^{11}(g)$ because $s$ meets $K^{11}$. But since $C g^{1} \Re T^{-1}(z)$ contains $\mathfrak{w}$, it follows that $g^{1}$ must belong to $K^{111}(g)$. Thus the proof is complete.

4.40. As a corollary to the preceding section, there follow the facts that the classes $K^{21}(g)$, for $g$ in $K^{0}$, and $K^{21}$ are finite. For consider the diagonally oriented square $s$ of 4.39 ; by $4.27(\zeta)$ it is true that $d$ is a subset of $h$. But then by $4.23(\delta)$ it is clear that $|h|$ is not less than $\delta_{0}^{2} / 8$. Since the elements of $K^{21}$ are mutually exclusive and are contained in the bounded set $\mathcal{D}$, it follows that $K^{21}$, and hence $K^{21}(g)$, are finite classes. 
4.41. For any set $h$ in $K^{21}$, the set $h T^{-1}(z)$ is a nonempty closed set contained in the bounded connected open set $2(h)$ (see $4.16,4.34,4.38$ ). Consequently there exists a finitely connected Jordan region $\mathfrak{r}(h)$ contained in $2(h)$ and containing $h T^{-1}(z)$ in its interior $\mathfrak{r}^{0}(h)$ (see 2.12). From 4.6 and 4.34 it follows that $\Re \mathfrak{r}(h)$ is a subset of $h$. By 4.39 it is clear that $\mathfrak{r}(h)$ has points in $K^{111}$. In fact, by using 4.31 and 4.39 , one may show that $\mathfrak{r}(h)$ may actually be chosen to lie in $h+K^{111} g$ (cf. 4.34), but this fact will not be needed in the sequel.

4.42. Denote by $M$ the class of all $\mathfrak{r}(h)$ for $h$ in $K^{21}$ (see 4.17). Of course, $M$ will be empty if $K^{21}$ is empty but this will not happen if $\Re$ contains points of $T^{-1}(z)$ (see 4.16). Using 4.4, 4.5, 4.15, 4.16, 4.34. 4.35, 4.36, 4.40, 4.41, the reader will deduce the following facts: $(\alpha) M$ is a finite class of mutually exclusive finitely connected Jordan regions in $\mathcal{D} ;(\beta) \mathcal{X}$ is a closed set in $\mathscr{D}$, and its interior $\mathscr{H}^{0}$ is given by $\sum \mathfrak{r}^{0}(h)$, the summation extending over those $h$ in $K^{21} ;(\gamma)$ for $g$ in $K^{01}$, the set $\mathcal{X} g$ is a closed set given by $\sum \mathfrak{r}(h)$, the summation extending over those $h$ in $K^{21}(g) ;(\delta) \Re \mathcal{X}$ is a subset of $K^{21} ;(\epsilon)$ these relations hold:

$$
C K^{2} T^{-1}(z)=K^{21} T^{-1}(z) \subset \mathcal{H}^{0} \subset \mathcal{A} \subset 2_{1} \subset K^{11}+K^{21} \subset K^{0} .
$$

4.43. The location of the set $T^{-1}(z)$ is summarized in the following relations.

$$
\begin{aligned}
\Re T^{-1}(z) & =K^{21} \Re T^{-1}(z) \subset \Re \mathcal{X}^{0} \subset \Re \Re \subset K^{21} ; \\
\mathcal{C} K^{21} T^{-1}(z) & =K^{11} T^{-1}(z)+K_{0} C K^{0} T^{-1}(z) .
\end{aligned}
$$

A proof follows readily from facts in $4.4,4.5,4.9,4.16,4.42$.

4.44. Now let $\Re_{1}$ and $\Re_{2}$ be two finitely connected oriented polygonal regions such that $\Re_{1}$ is contained in the interior $\Re_{2}^{0}$ of $\Re_{2}$, and $\Re_{2}$ is contained in $\mathcal{D}$. Subscripts 1 and 2 are added to the notations in troduced in $4.5,4.6,4.9$, $4.15,4.16,4.42$, to denote sets corresponding to $\Re_{1}$ and $\Re_{2}$ respectively, with $\tau, z, T, \mathcal{D}$, fixed as in 4.1 and 4.2 . Obviously $K_{1}^{0}$ is a subset of $K_{2}^{0}$, and $K_{1}^{01}$ is a subset of $K_{2}^{01}$-in fact, $K_{1}^{0}$ is a subset of $K_{2}^{0}$, and $K_{1}^{01}$ is a subset of $K_{2}^{01}$ (see 4.3).

4.45. The reader will have no difficulty in verifying that $K_{2}^{1} K_{1}^{0}$ is a subset of $K_{1}^{1}$, and $K_{2}^{11} K_{1}^{0}$ is a subset of $K_{1}^{11}$; but no similar relations exist amongst the othrer sets in 4.9. Then one readily sees that $K_{1}^{2}$ is a subset of $K_{2}^{2}$, and $K_{1}^{21}$ is a subset of $K_{2}^{21}$.

4.46. It will next be shown that one may assume $M_{2}$ so defined that $\mathscr{X}_{1}$ is a subset of $\mathcal{H}_{2}^{0}$ (see 4.42). From 4.40 and 4.45 it follows that each $h_{2}$ in $K_{2}^{21}$ contains a finite number-possibly none- of the $h_{1}$ in $K_{1}^{21}$. If $h_{2}$ does not contain any $h_{1}$ of $K_{1}^{21}$, let $\mathfrak{r}\left(h_{2}\right)$ be any Jordan region satisfying the conditions prescribed in 4.41. Otherwise, fix $\delta_{0}$ in 4.25 to be $\delta_{0}\left(\tau, T, \Re_{1}, \Re_{2}\right)$, and consider any $h_{1}$ contained in $h_{2}$. If $g$ denotes the element of $K_{2}^{01}$ containing $h_{2}$ (see 4.17), 
it follows from $4.15,4.33,4.34,4.41$, that $\mathfrak{r}\left(h_{1}\right) \subset 2\left(h_{1}\right)=h_{1}+K\left(h_{1}\right) \subset h_{2}+g \Re_{2}$ $\subset g \subset K_{2}^{11}$. Since $2\left(h_{1}\right)$ is connected, it follows that $r\left(h_{1}\right) \subset 2\left(h_{1}\right) \subset h_{2} \subset 2\left(h_{2}\right)$. Thus the sum $\mathcal{X}_{1} h_{2}$ of the $\mathfrak{r}\left(h_{1}\right)$ for $h_{1}$ in $h_{2}$ is a closed subset of $2\left(h_{2}\right)$, and a finitely connected Jordan region $\mathfrak{r}\left(h_{2}\right)$ may be chosen in $2\left(h_{2}\right)$ to contain the closed set $\mathfrak{x}_{1} h_{2}+h_{2} T^{-1}(z)$ in its interior $\mathfrak{r}^{0}\left(h_{2}\right)$ (see 2.12$)$. Clearly this $\mathfrak{r}\left(h_{2}\right)$ fulfills the requirements of 4.41 , and by using it in defining $M_{2}$, one obtains $\mathscr{X}_{1}$ as a subset of $\mathcal{O C}_{2}^{0}$.

4.47. Let $g$ be any element of $K_{1}^{0}$ and $h_{2}$ any element of $K_{2}^{2}(g)$ (see 4.16, 4.44). It is clear that $h_{2} K_{1}^{2}$ is a possibly empty set consisting of all $h_{1}$ in $K_{1}^{2}$ which are subsets of $h_{2}$. Moreover, it follows easily from 4.16 that $h_{2} K_{1}^{11}$ is the point set $h_{2} C K_{1}^{2}$. Let $f$ be any component of $h_{2} K_{1}^{11}=h_{2} C K_{1}^{2}$. Since $h_{2}$ is itself a component of $g C K_{2}^{11}$ (see 4.15), it follows that $f$ is a component of $g K_{1}^{11} C K_{2}^{11}$ (see Newman [1, IV]). Since $g K_{1}^{11}$ and $K_{2}^{11}$ are open sets (see 4.9), it follows from $4.18,4.19,4.32$, that $f$ fr $f=f C K_{2}^{11}=f C K_{2}^{11} \Re_{2} C \Re_{2}^{0}$, and $f^{0}=f \mathcal{C} C K_{2}^{11}=f h_{2}^{0}$.

4.48. Retain the assumptions of 4.47. Since $f$ is a connected subset of $K_{1}^{11} g$, there exists a $g_{1}^{1}$ in $K_{1}^{11}(g)$ which contains $f$ (see 4.6). Since $f$ is a component of $g K_{1}^{11} C K_{2}^{11}$ it follows that $f=C f g K_{1}^{11} C K_{2}^{11} \supset C f g_{1}^{1}$, and thus $g_{1}^{1}$ fr $f=f$ fr $f$ (see Newman [1, IV]). Now $f g_{1}^{1}$ reduces to $f$, which is not empty. If $\mathcal{C} f g_{1}^{1}$ is empty, it would follow that $f$ and $g_{1}^{1}$ are identical. But this is not possible, for $f T^{-1}(z)$ is a subset of the closed set $h_{2} T^{-1}(z)$ (see 4.38), while the set $C\left[g_{1}^{1} T^{-1}(z)\right]$ contains a point of $\mathcal{C D}$ (see 4.6). Thus $\mathcal{C} f g_{1}^{1}$ is not empty either. Since $g_{1}^{1}$ is connected it follows that $0 \neq g_{1}^{1}$ fr $f=f$ fr $f=f C K_{2}^{11}$ (see Newman [1, IV]; 4.47).

4.49. Retain the assumptions of 4.47 and 4.48. It will now be shown that there exists an element $g_{2}^{1}$ of $K_{2}^{11}$ such that $C g_{2}^{1} f$ contains a linear segment of points belonging to $\Re_{2} C \Re_{2}^{0}$. Let $\mathfrak{w}$ be any point of $f C K_{2}^{11}=f h_{2} g C K_{2}^{11} \Re_{2} C \Re_{2}^{0}$ $C g K_{1}^{11}$. Since $g K_{1}^{11}$ is open (see 4.9) there exists a diagonally oriented open square $s$ in $g K_{1}^{11}$ with center $\mathfrak{w}$ and diagonal length not exceeding $\delta_{0}$ (see 4.21,

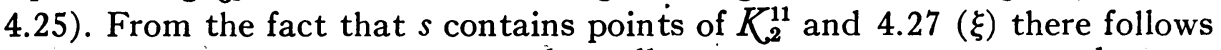
the existence of a unique element $g_{2}^{1}$ of $K_{2}^{11}$ such that $d_{0}$ is a subset of $g_{2}^{1}$. From $4.23(\gamma)$ and $4.27(\eta)$ it follows that $s \Re_{2} C \Re_{2}^{0}$ is a connected set in $h_{2} K_{1}^{11}$ containing the point $\mathfrak{w}$. Thus $s \Re_{2} C \Re_{2}^{0}$ is a subset of $C g_{2}^{1} f$ (see $4.27(\lambda)$ ), and the proof is complete.

4.50. Under the hypotheses used in $4.47,4.48,4.49$, it can be shown that the in terior $f^{\circ}$ of $f$ is also a connected set. A proof may be made along the lines of the proof in 4.31, using the general lemma in 4.30.

4.51. Now let $h_{1}$ be any element of $K_{1}^{2}$. A reasoning analogous to that in 4.48 and 4.49, with obvious simplifications, shows that there always exists an element $g_{1}^{1}$ of $K_{1}^{11}$ such that $C g_{1}^{1} h_{1}$ contains a linear segment of points belonging to $\Re_{1} \mathrm{C \Re}_{1}^{0}$. Of course, the same result is valid if the subscript 1 be replaced by 2 throughout.

4.52. Choose any sequence of finitely connected oriented polygonal re- 
gions $\mathfrak{r}_{n}$ in $\mathcal{D}$ such that $\mathfrak{r}_{n}$ is a subset of $\mathfrak{r}_{n+1}^{0}$ for every $n$ and the point set sum of the $\mathfrak{r}_{n}$ is $\mathscr{D}$ (see 2.12). Since $T^{-1}(z)$ is not empty (see 4.1) and $D$ contains a point not in $G$ (see 4.2 ), there exists a first in teger $n_{1}$ such that $\mathfrak{x}_{n_{1}}$-denote it by $\Re_{1}$ - contains a point of $T^{-1}(z)$ and a point not in $G$. A subscript 1 will be added to the notations in troduced in $4.5,4.6,4.9,4.15,4.16,4.42$, to denote sets corresponding to $\Re_{1}$ with $\tau, z, T, \mathcal{D}$ fixed as in 4.1 and 4.2. Since $\Re_{1}$ contains a point of $T^{-1}(z)$, the sets $K_{1}^{01}, K_{1}^{11}, K_{1}^{21}, M_{1}$, are not empty. Now $\Re_{1} T^{-1}(z)$ is a subset of $\Re_{1} \mathfrak{X}_{1}^{0}$ (see 4.43$)$; consequently $m_{1}^{1} \equiv m\left(t-z, \Re_{1} \mathcal{C X}_{1}^{0}\right.$ ) is a positive number (see 1.17). Put $\epsilon_{1}$ equal to $m_{1}^{1}$; observe that $\epsilon_{1}$ is a positive number less than $\tau$ since $\mathscr{X}_{1}$ is a nonempty subset of $G$ (see $4.42,4.5,4.2$ ).

4.53. Assume $\Re_{n-1}, \epsilon_{n-1}$ are defined. Since $\mathscr{X}_{n-1}$ is a closed set in $\mathcal{D}$ (see 4.42), there exists a first in teger $n_{n}$ greater than $n_{n-1}$ such that $\mathfrak{r}_{n_{n}}$-denote it by $\Re_{r}$-contains $\mathscr{X}_{n-1}$ in its interior $\Re_{n}^{0}$. A subscript $n$ will be added to the notations introduced in $4.5,4.6,4.9,4.15,4.16,4.42$, to denote sets corresponding to $\Re_{n}$ with $\tau, z, T, \mathcal{D}$, fixed as in 4.1 and 4.2. Assume further that $M_{n}$ is so chosen that $\mathcal{X}_{n-1}$ is a subset of $\mathscr{X}_{n}$ (see 4.46). Clearly the sets $K_{n}^{01}$, $K_{n}^{11}, K_{n}^{21}, M_{n}$ are not empty (see $4.44,4.45$ ). Since $\Re_{n} T^{-1}(z)$ is a subset of $\Re_{n} \mathcal{X}_{n}^{0}$ (see 4.43), the constant $m_{n}^{n}=m\left(t-z, \Re_{n} \mathcal{C} \mathcal{X}_{n}^{0}\right)$ is a positive number. For any positive integer $j$ less than $n$, the set $C K_{j}^{2} \Re_{n} T^{-1}(z)$ is a subset of $\mathcal{X}_{j}^{0}$

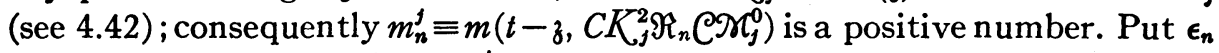
equal to the smallest of the $m_{n}^{j}$ for positive integers $j$ not exceeding $n$. Then $\epsilon_{n}$ is a positive number less than $\tau$. The definitions of the $\Re_{n}$ and the $\epsilon_{n}$ are completed by induction. Observe that $\sum_{n} \Re_{n}=\sum_{n} \Re_{n} \mathcal{C} \Re_{n-1}^{0}=\mathcal{D}$, where $\Re_{0}$ is defined to be the empty set (see 2.12).

4.54. By the approximation lemma in 2.14 , there exists a quasi-polyhedral transformation $T_{*}: z=t_{*}(w)$, weD, such that the distance $\rho\left(T_{*}, T, \Re_{n} \mathcal{C} \Re_{n-1}^{0}\right)$ is less than $\epsilon_{n}$ for every $n$, and $T_{*}^{-1}(z)$ has no points in $\sum_{n} \Re_{n} \mathcal{C} \Re_{n}^{0}$ (see 2.16). Since the $\epsilon_{n}$ are less than $\tau$ (see 4.53), it is clear that $\rho\left(T_{*}, T, \mathscr{D}\right)$ does not exceed $\tau$. The location of $T_{*}^{-1}(z)$ is presently discussed.

4.55. For every positive integer $n$, the set $\Re_{n} T_{*}^{-1}(z)$ is contained in $\mathscr{H}_{n}^{0}$. This follows at once from these inequalities (see 4.53, 4.54):

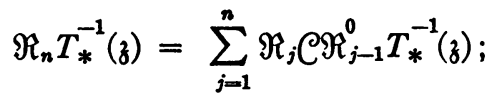

$$
\begin{aligned}
& m\left(t_{*}-z, \Re_{j} \mathcal{C} \Re_{j-1}^{0} \mathcal{C} \mathcal{X}_{n}^{0}\right) \geqq m\left(t_{*}-z, \Re_{j} \mathcal{C} \Re_{j-1}^{0} \mathcal{C} \mathcal{X} x_{j}^{0}\right) \\
& \geqq m\left(t-z, \Re_{j} \mathcal{C} \mathcal{X}_{j}^{0}\right)-M\left(t_{*}-t, \Re_{j} \mathcal{C} \Re_{j-1}^{0}\right) \\
& >m_{j}^{j}-\epsilon_{j} \geqq 0, \quad j=1, \cdots, n \text {. }
\end{aligned}
$$

From $4.42(\delta)$ it now follows that $\Re_{n} T_{*}^{-1}(z)$ is a subset of $K_{n}^{21}$.

4.56. For every positive integer $n$, and every positive integer $j$ for which $j-1$ exceeds $n$, the set $K_{n}^{2} \Re_{j} \mathcal{C} \Re_{j-1}^{0} T_{*}^{-1}(z)$ is empty. A proof is contained in the inequalities following (see $4.53,4.54$ ). 


$$
\begin{aligned}
m\left(t_{*}-z_{1}, K_{n}^{2} \Re_{j} \mathcal{C} \Re_{j-1}^{0}\right) & =m\left(t_{*}-z, K_{n}^{2} \Re_{j} \mathcal{C} \Re_{j-1}^{0} \mathcal{C} \mathcal{X}_{n}^{0}\right) \\
& \geqq m\left(t-z, C K_{n}^{2} \Re_{j} \mathcal{C} \mathcal{X}_{n}^{0}\right)-M\left(t_{*}-t, \Re_{j} \mathcal{C} \Re_{j-1}^{0}\right) \\
& >m_{j}^{n}-\epsilon_{j} \geqq 0 .
\end{aligned}
$$

Consequently (see 4.54 ) one obtains these relations:

$$
K_{n}^{2} T_{*}^{-1}(\mathrm{z})=K_{n}^{2} T_{*}^{-1}(\mathrm{z}) \sum_{j=1}^{n+1} \Re_{j} \mathcal{C} \Re_{j-1}^{0} \subset T_{*}^{-1}(\mathrm{z}) \Re_{n+1} \subset \Re_{n+1}^{0}
$$

4.57. The set $K_{n}^{2} \Re_{n+1} \mathcal{C} \Re_{n}^{0} T_{*}^{-1}(z)$ is contained in $\mathcal{H}_{n}^{0}$ for every integer $n$. This is a consequence of these inequalities (see 4.53, 4.54).

$$
\begin{aligned}
m\left(t_{*}-z_{1}, K_{n}^{2} \Re_{n+1} \mathcal{C} \Re_{n}^{0} \mathcal{C} x_{n}^{0}\right) & \geqq m\left(t-z, C K_{n}^{2} \Re_{n+1} \mathcal{C} \mathcal{X}_{n}^{0}\right)-M\left(t_{*}-t, \Re_{n+1} \mathcal{C} \Re_{n}^{0}\right) \\
& >m_{n+1}^{n}-\epsilon_{n+1} \geqq 0 .
\end{aligned}
$$

From 4.55 and 4.56 it follows that $K_{n}^{2} T_{*}^{-1}(z)$ must be contained in $\mathcal{X}_{n}^{0}$.

4.58. Let $h_{n}$ be any element of $K_{n}^{2}$. From $4.16,4.19,4.31,4.54$, it follows easily that $h_{n} T_{*}^{-1}(z)$ is a subset of the connected interior $h_{n}^{0}$ of $h_{n}$. Moreover, if $f_{n}$ be any component of $h_{n} K_{n-1}^{11}=h_{n} \mathcal{C} K_{n-1}^{2}$ (see 4.47), it follows that $f_{n} T_{*}^{-1}(z)$ is a subset of the connected interior $f_{n}^{0}$ of $f_{n}$ (see 4.50).

4.59. $T_{*}$ will now be modified. From 4.57 it is clear that $K_{1}^{2} T_{*}^{-1}(z)$ is contained in $\mathscr{H}_{1}^{0}$, and hence the number $N\left(z, T_{*}, K_{1}^{2}\right)$ is finite (see $4.53,4.54$, 2.13). Let $K_{* 1}$ denote the class of all elements $h_{1}$ in $K_{1}^{2}$ which contain points in $T_{*}^{-1}(z)$ (see 4.16); this is obviously a finite class. It is readily seen that $K_{1}^{2} T_{*}^{-1}(z)$ is contained in $K_{* 1}^{0}$ (see $\left.4.3,4.58\right)$. In view of $4.31,4.51$, it now follows that, for each $h_{1}$ in $K_{* 1}$, there is a Jordan region $\Re\left(h_{1}\right)$ possessing the following properties: (i) $\Re\left(h_{1}\right)$ is simply connected; (ii) $h_{1} T_{*}^{-1}(z)$ is contained in $\Re^{0}\left(h_{1}\right)$ and $\Re\left(h_{1}\right)$ is contained in $h_{1}+K_{1}^{11} \Re_{2}^{0}$; (iii) if $h_{1}$ and $h_{1}^{\prime}$ are two different elements of $K_{* 1}$, then $\Re\left(h_{1}\right)$ has no points in common with either $\Re\left(h_{1}^{\prime}\right)$ or $h_{1}^{\prime}$; (iv) $\Re\left(h_{1}\right) \mathcal{C} \Re^{0}\left(h_{1}\right)$ contains no points of $T_{*}^{-1}(z)$; (v) there is a point $\mathfrak{w}\left(h_{1}\right)$ in $K_{1}^{11} \Re^{0}\left(h_{1}\right)$. Denote by $L_{1}$ the set of points $\mathfrak{w}\left(h_{1}\right)$ for $h_{1}$ in $K_{* 1}$; observe that $L_{1}$ is a finite set. Let $M_{* 1}$ denote the class of Jordan regions $\Re\left(h_{1}\right)$ for $h_{1}$ in $K_{* 1}$; this is a finite class of mutually exclusive Jordan regions in $G$ (see 4.2 ). Using $4.9,4.16,4.44$, the reader will verify the following relations.

$$
\begin{gathered}
K_{1}^{2} T_{*}^{-1}(z) \subset \mathcal{X}_{* 1}^{0} \subset \mathcal{X}_{* 1} \subset K_{* 1}+K_{2}^{2} \subset K_{1}^{2} K_{1}^{11} \Re_{2}^{0} \subset \Re_{1} \\
\subset K_{1}^{2}+K_{2}^{2} \subset K_{1}^{2} K_{1}^{11} \Re_{2}^{0} \subset \Re_{1} \subset G K_{2}^{2} ; \\
\mathcal{L}_{1} \subset K_{1}^{11} \mathcal{X}_{* 1}^{0} \subset K_{2}^{2} \subset K_{1}^{2} K_{1}^{11} \Re_{2}^{0} \subset \Re_{1} .
\end{gathered}
$$

4.60. Define $t_{1}(w)$ to be $t_{*}(w)$ for $w$ in $\mathscr{D} \mathcal{C} \mathfrak{X}_{* 1}$. For each $\Re\left(h_{1}\right)$ in $M_{* 1}$, the hypotheses of the corollary in 3.5 are evidently satisfied by $\Re\left(h_{1}\right), \mathfrak{w}\left(h_{1}\right)$, $T_{*}, z$ (see 4.59); thus it is possible to modify $t_{*}(w)$ in each $\Re\left(h_{1}\right)$ in a continuous way to obtain a continuous function $t_{1}(w)$ having the properties described 
in 3.5. The transformation $T_{1}: z=t_{1}(w), w \in \mathcal{D}$, has the following properties: $(\alpha)$ Clearly $T_{1}$ is a bounded continuous transformation. $(\beta)$ Obviously $T_{1}$ reduces to $T_{*}$ on $\mathcal{D} \mathcal{C} \mathcal{P} r_{* 1}^{0}$ which contains $\mathcal{C} K_{2}^{2}$. $(\gamma) N\left(z, T_{1}, K_{1}^{2}\right)=0$; to see this, consider any $h_{1}$ in $K_{1}^{2}$. If $h_{1}$ is not in $K_{* 1}$ then it follows from $4.59(\alpha)$ that $h_{1}$ is in $\mathcal{C} \mathscr{X}_{* 1}$ and consequently, in view of $(\beta), N\left(z, T_{1}, h_{1}\right)$ is zero. If $h_{1}$ is in $K_{* 1}$,

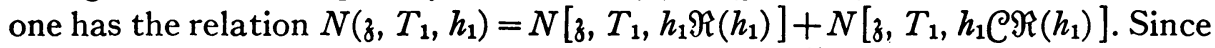
the only model of $z$ under $T_{1}$ in $\Re\left(h_{1}\right)$ is $\mathfrak{w}\left(h_{1}\right)$ in $K_{1}^{11}$ (see $4.59(\beta)$ ), it is clear that the first term of this sum is zero (see 4.16). Now $h_{1} \varrho \Re\left(h_{1}\right)$ is a subset of

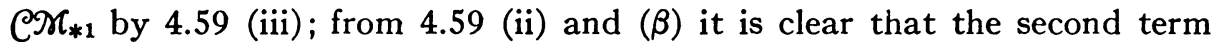
of this sum is also zero. Thus $N\left(z, T_{1}, h_{1}\right)$ is zero, and $(\gamma)$ holds. $(\delta) N\left(z, T_{1}, \Re_{1}\right)$ $=0$; this may be seen as follows. In view of $(\gamma)$ one has the relation $N\left(z, T_{1}, \Re_{1}\right)=N\left(z, T_{1}, \mathcal{C} K_{1}^{2} \Re_{1}\right)$. But $\Re_{1} \mathcal{C} K_{1}^{2}$ is a subset of $\mathcal{C} \mathcal{X}_{* 1}$ (see $\left.4.59(\alpha)\right)$;

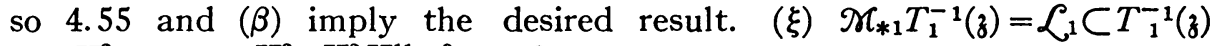

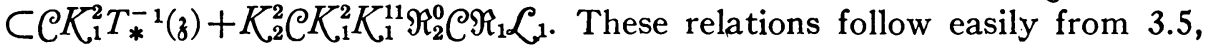
4.59 , and $(\beta)$. $(\eta) M\left(t_{1}-z, G\right) \leqq 2 \tau$; to show this, write $G=G \mathcal{C} \mathcal{X}_{* 1}+\mathcal{X}_{* 1}$. In view of $4.2,4.54$, and $(\beta)$ it is clear that $M\left(t_{1}-z, G C \mathcal{X}_{* 1}\right)$ does not exceed $2 \tau$. Consider any $\Re\left(h_{1}\right)$ in $M_{* 1}$; from 3.5 it follows at once that $M\left[t_{1}-z, \Re\left(h_{1}\right)\right]$ is less than $2 \tau$. Since the elements of $M_{* 1}$ are mutually exclusive, it follows that $M\left(t_{1}-\xi, \mathcal{X}_{* 1}\right)$ does not exceed $2 \tau$, and the result is established. $(\zeta) \rho\left(T_{1}, T, \mathscr{D}\right)<3 \tau$; this may be shown as follows. Write $\mathcal{D}=\mathscr{D} \mathcal{C} \mathcal{X}_{* 1}+\mathcal{X}_{* 1}$. From 4.54 and $(\beta)$ it is clear that $\rho\left(T_{1}, T, \mathscr{D} \mathcal{C \mathcal { X } _ { * 1 } )}\right.$ does not exceed $\tau$. On the other hand, $M\left(t_{1}-t, \mathcal{X}_{* 1}\right)$ is not greater than $M\left(t_{1}-z, \mathcal{X}_{* 1}\right)+M\left(t-z, \mathcal{X}_{* 1}\right)$, which is less than $3 \tau$ by $4.2,4.54$, and $(\eta)$.

4.61. Next, assume that finite sets $K_{* n-1}, L_{n-1}, M_{* n-1}$, and a transformation $T_{n-1}$ have been defined so that the following conditions hold: $(\alpha) T_{n-1}$ is a bounded continuous transformation. $(\beta) T_{n-1}$ reduces to $T_{n-2}$ on $\mathcal{D} \mathcal{C} \mathcal{X}_{* n-1}^{0}$; $T_{n-1}$ reduces to $T_{j}$ on $\Re_{j}+K_{j}^{2}$ for positive integers $j$ less than $n-1 ; T_{n-1}$ reduces to $T_{*}$ on $\mathcal{D} \prod_{j=1}^{n-1} \mathcal{C} \mathcal{X K}_{* j}^{0}$, which contains $\mathcal{C} K_{n}^{2}$. $(\gamma) N\left(z, T_{n-1}, K_{n-1}^{2}\right)=0$;

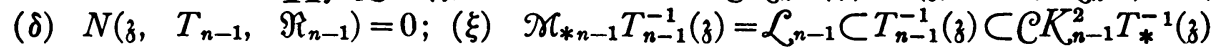

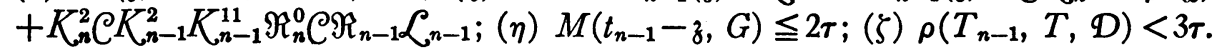
Presently $K_{* n}, L_{n}, M_{* n}, T_{n}$ will be defined to satisfy these same relations.

4.62. From 4.57 it is clear that $K_{n}^{2} T_{*}^{-1}(z)$ is contained in $\mathcal{H}_{n}^{0}$, which is a subset of $\Re_{n+1}^{0}$ by 4.53. From 4.61 ( $\left.\xi\right)$ it follows that $K_{n}^{2} T_{n-1}^{-1}(z)$ is contained in $K_{n}^{2} \mathrm{C} K_{n-1}^{2} T_{*}^{-1}(z)+K_{n}^{2} \mathrm{C} K_{n-1}^{2} \mathcal{L}_{n-1}$; thus $N\left(z, T_{n-1}, K_{n}^{2}\right)$ must be finite (see 2.13). Let $K_{* n}$ denote the class of all components $f_{n}$ of $h_{n} C K_{n-1}^{2}$, where $h_{n}$ belongs to $K_{n}^{2}$, which contain a point of $T_{n-1}^{-1}(z)$ (see 4.47); this is obviously a finite class. It is readily seen that $K_{n}^{2} T_{n-1}^{-1}(z)$ is contained in $K_{* n}^{0}$ (see 4.3, $4.47,4.58)$. For if $\mathfrak{w}$ be any point in $K_{n}^{2} T_{n-1}^{-1}(z)$ there exists an $h_{n}$ of $K_{n}^{2}$ containing $\mathfrak{w}$; let $f_{n}$ be the component of $h_{n} C K_{n-1}^{2}$ which contains $\mathfrak{w}$. In view of the above relations, it is clear that either $\mathfrak{w}$ belongs to $T_{*}^{-1}(z)$ or $\mathfrak{w}$ belongs to $\mathcal{L}_{n-1}$. If $\mathfrak{w}$ belongs to $T_{*}^{-1}(z)$, then $\mathfrak{w}$ belongs to $h_{n}^{0} f_{n}$, or $f_{n}^{0}$, by $4.47,4.58$. If $\mathfrak{w}$ belongs to $\mathcal{L}_{n-1}$, it follows, because $\mathcal{L}_{n-1}$ is a subset of $\Re_{n}^{0}$, that w belongs to $f_{n} \mathcal{C} C K_{n}^{11}$, or $f_{n}^{0}($ see $4.9,4.47)$. So it is seen that $K_{n}^{2} T_{n-1}^{-1}(z)$ is a subset of $K_{* n}^{0}$. 
In view of $4.49,4.50$, it now follows that, for each $f_{n}$ in $K_{*_{n}}$, there is a Jordan region $\Re\left(f_{n}\right)$ possessing the following properties: (i) $\Re\left(f_{n}\right)$ is simply connected; (ii) $f_{n} T_{n-1}^{-1}(z)$ is contained in $\Re^{0}\left(f_{n}\right)$ and $\Re\left(f_{n}\right)$ is contained in $f_{n}+K_{n}^{11} \Re_{n+1}^{0}$; (iii) if $f_{n}$ and $f_{n}^{\prime}$ are two different elements of $K_{* n}$ then $\Re\left(f_{n}\right)$ has no points in common with either $\Re\left(f_{n}^{\prime}\right)$ or $f_{n}^{\prime}$; (iv) $\Re\left(f_{n}\right) \mathcal{C} \Re^{0}\left(f_{n}\right)$ contains no points of $T_{n-1}^{-1}(z)$; (v) there is a point $\mathfrak{w}\left(f_{n}\right)$ in $K_{n}^{11} \Re^{0}\left(f_{n}\right)$. Denote by $L_{n}$ the set of points $\mathfrak{w}\left(f_{n}\right)$ for $f_{n}$ in $K_{* n}$; observe that $L_{n}$ is a finite set. Let $M_{* n}$ denote the class of Jordan regions $\Re\left(f_{n}\right)$ for $f_{n}$ in $K_{* n}$; this is a finite class of mutually exclusive Jordan regions in $G$ (see 4.2). Using 4.5, 4.9, 4.16, 4.44, 4.45, the reader will verify the following relations.

$$
\begin{aligned}
& K_{n}^{2} T_{n-1}^{-1}(\S) \subset \mathcal{X}_{* n}^{0} \subset \mathcal{X}_{* n} \subset K_{* n}+K_{n+1}^{2} \subset K_{n}^{2} K_{n}^{11} \Re_{n+1}^{0} \bigodot \Re_{n}
\end{aligned}
$$

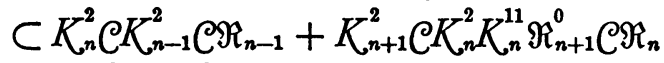

$$
\begin{aligned}
& \subset G K_{n+1}^{2} \odot K_{n-1}^{2} \odot \Re_{n-1} \text {; }
\end{aligned}
$$

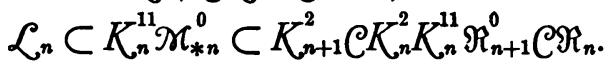

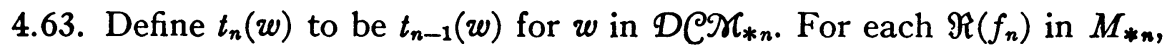
the hypotheses of the corollary in 3.5 are evidently satisfied by $\Re\left(f_{n}\right), \mathfrak{w}\left(f_{\mathfrak{n}}\right)$, $T_{n-1}, z$ (see 4.62); thus it is possible to modify $t_{n-1}(w)$ in each $\Re\left(f_{n}\right)$ in a continuous way to obtain a continuous function $t_{n}(w)$ having the properties described in 3.5. It remains to verify that the transformation $T_{n}: z=t_{n}(w), w \in \mathcal{D}$, has the properties $(\alpha)-(\zeta)$ of 4.61. $(\alpha)$ Clearly $T_{n}$ is a bounded continuous transformation. $(\beta)$ That $T_{n}$ reduces to $T_{n-1}$ on $\mathscr{D} \mathcal{C} \mathcal{S}_{* n}^{0}$ follows from the definition of $T_{n}$. From $4.62(\alpha)$ it is evident that $\Re_{n-1}+K_{n-1}^{2}$ is a subset of $\mathcal{C} \mathcal{X}_{* n}^{0}$, so $T_{n}$ reduces to $T_{n-1}$ on $\Re_{n-1}+K_{n-1}^{2}$. For positive integers $j$ less than $n-1$, the set $\Re_{j}+K_{j}^{2}$ is a subset of $\Re_{n-1}+K_{n-1}^{2}$ (see $4.45,4.52$ ), so $T_{n}$ reduces to $T_{j}$ on $\Re_{j}+K_{\text {j }}^{2}$ by $4.61(\beta)$. Again, by $4.61(\beta)$ it is clear that $T_{n}$ reduces to $T_{*}$ on $\mathcal{D} \prod_{j-1}^{n} \mathcal{C} \mathcal{X}_{* j}^{0}$; the fact that this set contains $\mathcal{C} K_{n+1}^{2}$ may be verified by using $4.45,4.62(\alpha)$, and $4.61(\beta) .(\gamma) N\left(z, T_{n}, K_{n}^{2}\right)=0$; to see this, consider the relation (see 4.45) $N\left(z, T_{n}, K_{n}^{2}\right)=N\left(z, T_{n}, K_{n}^{2} C K_{n-1}^{2}\right)+N\left(z, T_{n}, K_{n-1}^{2}\right)$. From $4.61(\gamma)$ and $(\beta)$ it is clear that the second term of this sum is zero. That the first term of this sum is also zero may be shown by a reasoning similar to that in $4.60(\gamma) .(\delta) N\left(z, T_{n}, \Re_{n}\right)=0$. A reasoning similar to that in $4.60(\delta)$ shows that $N\left(z, T_{n}, \Re_{n}\right)$ has the same value as $N\left(z, T_{n-1}, \mathcal{C} K_{n}^{2} \Re_{n}\right)$.

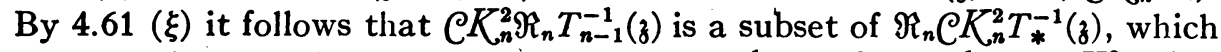
is empty by 4.55 . Thus $(\delta)$ holds. $(\xi) \mathcal{X}_{* n} T_{n}^{-1}(z)=\mathcal{L}_{n} \subset T_{n}^{-1}(z) \subset \subset K_{n}^{2} T_{*}^{-1}(z)$ $+K_{n+1}^{2} \mathcal{C} K_{n}^{2} K_{n n}^{11} \Re_{n+1}^{0} \mathcal{C} \Re_{n} \mathcal{K}_{n}$. These relations follow easily from 3.5, $4.61(\beta)$, 4.62 , and $(\beta) .(\eta) M\left(t_{n}-z, G\right) \leqq 2 \tau$; this may be shown by a reasoning similar to that in $4.60(\eta)$, using $3.5,4.61(\eta)$, and $(\beta) .(\zeta) \rho\left(T_{n}, T, \Phi\right)<3 \tau$; this follows by an argument analogous to that in $4.60(\zeta)$, using 4.2, $4.61(\zeta)$, and $(\beta),(\eta)$ of this section. Thus the definitions of the $K_{* n}, L_{n}, M_{* n}, T_{n}$, are complete by induction. 
4.64. For each $\mathfrak{w}$ in $\mathcal{D}$ there is a first positive integer $n$ such that $\mathfrak{w}$ is in $\Re_{n}$ (see $4.52,4.53,2.12)$. Define $t_{f}(\mathfrak{w})$ to be $t_{n}(\mathfrak{m})$ for $\mathfrak{w}$ in $\mathcal{D}$. The reader will have no difficulty in verifying that the transformation $T_{\sharp}: z=t_{f}(w), w \in \mathcal{D}$, has the following properties (see 4.63): $(\alpha) T_{f}$ is a bounded continuous transformation; $(\beta) T_{f}$ is identical with $T_{n}$ on $\Re_{n}$ for every positive integer $n ;(\gamma) z$ has no models under $T_{f}$ in $\mathcal{D}$-that is, $N\left(z, T_{\sharp}, \mathcal{D}\right)=0 ;(\delta) \rho\left(T_{f}, T, \mathcal{D}\right)$ does not exceed $3 \tau$.

4.65. The results obtained in this chapter may be summarized as follows.

TheOREM. Let $\mathcal{D}$ be a bounded domain in the w-plane. Given a bounded continuous transformation $T: z=t(w), w \in \mathcal{D}$, let $z$ be any point of the z-plane for which no component of $T^{-1}(z)$ is a continuum. If $\epsilon$ be any positive number, there exists a bounded continuous transformation $T_{\sharp}: z=t_{f}(w), w \in \mathcal{D}$, such that the distance $\rho\left(T_{\sharp}, T, \mathcal{D}\right)$ is less than $\epsilon$, and $z$ has no models under $T_{\neq}$in $\mathcal{D}$.

Thus if no component of $T^{-1}(z)$ is a continuum, the question raised in 1.10 is now answered.

\section{Chapter V. A proof of the theorem}

5.1. A proof for the theorem stated in 1.11 is now made. Let $\mathcal{D}$ be any bounded domain in the $w$-plane. Suppose $T: z=t(w), w \in \mathcal{D}$, is a bounded continuous transformation (see 1.2). Assume $z$ is any point of the $z$-plane and $\epsilon$ is any positive number. It is convenient to consider five cases: $(\alpha)$ If the essential multiplicity $\kappa(z, T, \mathcal{D})$ is infinite (see $\mathrm{R}^{2}[1,1.13]$ ), the theorem is obvious - in fact, one has a quasi-polyhedral transformation $T_{t}: z=t_{\boldsymbol{t}}(w), w \in \mathcal{D}$, which not only possesses the properties required in the theorem, but also enjoys the additional properties described in 2.13 (see $2.14,2.16$ ). ( $\beta$ ) If the number of models of $z$ under $T$ in $\mathcal{D}, N(z, T, \mathcal{D})$, is zero (see $\mathrm{R}^{2}[1,1.10]$ ), the theorem is trivial. $(\gamma)$ If every component of $T^{-1}(z)$ is a continuum (see 3.1 ), the theorem is a consequence of 3.13. ( $\delta$ ) If no component of $T^{-1}(z)$ is a continuum, the theorem is a consequence of 4.65 . $(\epsilon)$ There remains only the case where the essential multiplicity $\kappa(z, T, \mathcal{D})$ has a finite value $k$, and some of the components of $T^{-1}(z)$ are continua, others are not.

5.2. According to the theorem in 3.13 , there exists a bounded continuous transformation $T_{1}: z=t_{1}(w)$, weD, such that the distance $\rho\left(T_{1}, T, \mathscr{D}\right)$ is less than $\epsilon / 3$, and the set $T_{1}^{-1}(z)$ consists of precisely $k$ isolated points $\mathfrak{m}$ and the totality of components of $T^{-1}(z)$ which are not continua. Let $L$ denote the class consisting of the $k$ isolated points $\mathfrak{w}$ in $T_{\mathbf{1}}^{-1}(\mathfrak{z})$. Choose a class $M$ of $k$ mutually exclusive oriented closed squares $s$ in $\mathcal{D}$ with centers $\mathfrak{w}$ in $L$, such that $s \cdot T_{1}^{-1}(\mathfrak{z})=\mathfrak{w}$. Now pick a class $M_{1}$ of $k$ oriented closed squares $S$ each of which is concentric with and contained in the interior of the corresponding square $s$ in $M$. Evidently (see 4.3), $\mathscr{X} 1_{1}$ is a subset of $\mathcal{X} \mathcal{X}^{0}$, and the sets $\mathcal{X} T_{1}^{-1}(z)$ and $\mathscr{H}_{1}^{0} T_{1}^{-1}(z)$ both reduce to $\mathcal{L}$. Consequently $m=m\left(t_{1}-z, \mathcal{H} \mathcal{C} \mathcal{H}_{1}^{0}\right)$ is a positive number. 
5.3. Clearly $\mathscr{D} \mathcal{C} \mathcal{L}$ is a bounded domain $\mathscr{D}_{1}$ in $\mathscr{D}$ (see Newman [1, IV]). Consider the transformation $T_{11}: z=t_{11}(w) \equiv t_{1}(w), w \in \mathcal{D}_{1}$. Evidently $T_{11}$ is a bounded continuous transformation for which no component of $T_{11}^{-1}(z)$ is a continuum. From 4.65 it follows that there exists a bounded continuous transformation $T_{12}: z=t_{12}(w)$, $w \in \mathscr{D}_{1}$, for which the distance $\rho\left(T_{12}, T_{11}, \mathscr{D}_{1}\right)$ is less than either of $\epsilon / 3$ or $m$ (see 5.2), and the number of models of $z$ under $T_{12}$ in $\mathscr{D}_{1}, N\left(\mathfrak{z}, T_{12}, \mathscr{D}_{1}\right)$, is zero.

5.4. Now consider any $s$ in $M$ and its corresponding $S$ in $M_{1}$ (see 5.2). Clearly $m\left(t_{1}-z, S C S^{0}\right)$ exceeds $M\left(t_{1}-t_{12}, S C S^{0}\right)$. Thus $z=t_{1}(w)$, $w \in S$, may be

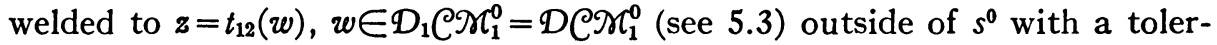
ance of $\epsilon / 3$ and the exclusion of $z$ in the image of $s \mathcal{C} S^{\circ}$ (see 2.11). Denote the transformation resulting from the application of 2.11 to each $s$ in $M$, together with its corresponding $S$ in $M_{1}$, by $T_{f}: z=t_{f}(w), w \in \mathcal{D}$. The reader will have no difficulty in verifying that $T_{*}$ has the properties required in the theorem in 1.11 .

5.5. Since the essential multiplicity $\kappa(z, T, D)$ is a lower semi-continuous integral-valued function of $T$ (see $\mathrm{R}^{2}[1,2.18]$ ), there exists a positive number $\zeta$ such that $\kappa\left(z, T_{*}, \mathcal{D}\right)$ is not less than $k$ for any bounded continuous transformation $T_{*}: z=t_{*}(w), w \in \mathcal{D}$, whose distance $\rho\left(T_{*}, T, \mathcal{D}\right)$ from $T$ on $\mathcal{D}$ is less than $\zeta$. If $\epsilon$ in 5.1 be less than $\zeta$, it is clear that each of the $k$ models of $z$ under $T_{f}$ in $\mathscr{D}$ must constitute an essential maximal model continuum for $z$ un$\operatorname{der} T_{\boldsymbol{f}}$ in $\mathcal{D}$ (see $\left.\mathrm{R}^{2}[1,1.16,1.17]\right)$.

\section{BIBLIOGRAPHY}

S. BANACH

1. Sur les lignes rectifiables et les surfaces dont l'aire est finie, Fund. Math. vol. 7 (1925) pp. 225-236.

\section{R. L. MOORE}

1. Foundations of point set theory, Amer. Math. Soc. Colloquium Publications, vol. 13, 1932.

M. H. A. Newman

1. Elements of the topology of plane sets of points, Cambridge, 1939.

T. RAD6

1. Über das Flächemmass rektifizierbarer Flachen, Math. Ann. vol. 100 (1928) pp. 445-479.

2. On absolutely continuous transformations in the plane, Duke Math. J. vol. 4 (1938) pp. 189-221.

T. RADó and P. Reichelderfer

1. A theory of absolutely continuous transformations in the plane, Trans. Amer. Math. Soc. vol. 49 (1941) pp. 258-307.

P. V. REICHELDERFER

1. On bounded variation and absolute continuity for parametric representations of continuous surfaces, Trans. Amer. Math. Soc. vol. 53 (1943) pp. 251-291.

\section{J. SCHAUDER}

1. Über stetige Abbildungen, Fund. Math. vol. 12 (1928) pp. 47-74.

UNIVERSITY OF Wisconsin, Madison, Wis. 